\title{
Optimal Protection Index in Malaria Vector Hosts Elicited by the 10-34 kHz Animal Sound
}

\author{
Mang'are P. A. ${ }^{1,2 *}$, Ndiritu F. G. ${ }^{1}$, Rotich S. K. ${ }^{3}$, MakatianiJ. K., ${ }^{4}$ Rapando B.W. ${ }^{2}$ \\ ${ }^{1}$ Physics Department,EgertonUniversity. P.O Box 536-20115, Egerton, Njoro. Kenya \\ ${ }^{2}$ Physics Department,Masinde Muliro Universityof Science and Technology. P. O Box 190-50100, Kakamega. Kenya \\ ${ }^{3}$ Department of Physicsand Mathematics., Moi University, P. O. Box 3900-30100 Eldoret. Kenya \\ ${ }^{4}$ Department of BiologicalSciences., Moi University, P. O. Box 3900-30100 Eldoret. Kenya
}

\begin{abstract}
A coustic startle has been exploited in the control of malaria by targeting the female Anopheles gambiae. Studies with an inbuilt ultrasonic device (AC-UD) yielded $17.3 \%$ and $60.7 \%$ in the knockdown tests with fan-off and fan-on respectively. The repellency by the 10-34 kHz sound of Odorrana tormota based on observable behavioural responses and the unconfirmed repellency due to the sound from Anti-Pic ${ }^{\circledR}$ (EMR) on the female A. gambiae was $34.12 \%$ and $30.3 \%$ respectively. Recent studies with the electronic piezo buzzer mosquito repellent emitting $40-55 \mathrm{kHz}$ sound treatment on mosquitoes yielded a protection index of $68.99 \%$. Chemical malaria interventions were impeded by pathogen and vector resistance. This research thus determined and analyzed the optimal protection index in malaria vector hosts based on landing rates and bites elicited by the 10-34 kHz filtered recorded sounds of the male Anopheles gambiae, mixed male and female Delphinapterus leucas, and further investigated the sound of the male 0 . tormota. Landing rates and behavioral responses of the female A. gambiae which were bred and reared under controlled laboratory conditions in Kenya Medical Research Institute evoked by the filtered into $10-34 \mathrm{kHz}$ sound of the male mosquito, A. gambiae, male 0 . tormota, and mixed male and female $D$. leucas were determined and analyzed statistically. The sounds of the A. gambiae, 0 . tormota, and D. leucas yielded 2.10, 2.20, and 3.00 landings (bites)/minute; and 42.73\%, $40.24 \%$, and $10.64 \%$ protection index respectively. The optimal acoustic entropy, power deviation, and average acoustic power of the sound of the male $A$. gambiae were 4.58 bits, $35.80 \mathrm{~dB}$, and $51.60 \mathrm{~dB}$ respectively with wide bandwidth. The protection index evoked by the sound of the male A. gambiae did not differ significantly from the reported repellency of the sound of $O$. tormota and Anti-Pic $®$ EMR, though differed significantly from the sound emitted by the and AC-UD.
\end{abstract}

Keywords Protection index; Electronic Mosquito Repellent; Malaria; Bandwidth; Pulsate

\section{INTRODUCTION}

\section{Worldwide Malaria Situation and Interventions}

Malaria causes adverse effects including low birth weights, impaired physical growth, and permanent disability [80]. Notably, the challenge of malaria causes substantial costs to both individuals and governments, with estimated global financing increasing from the US \$1.3 billion in 2017 to the US $\$ 2.3$ billion in 2018 [82]. Adherence to recommended interventions renders malaria a preventable and treatable disease [76, 78, 81]. Therefore, minimizing the host-vector interactions has been viewed as an effective way of reducing malaria $[66,82]$.

\section{Chemical prevention, treatment, and control of malaria}

The chemoprevention is used for the most vulnerable populations, particularly pregnant women and infants. Confirmation of malaria diagnosis through microscopy or rapid diagnostic tests (RDTs) for every suspected 
case and timely treatment with appropriate antimalarial medicines is key in malaria control $[16,28,31,35$, $78,79,81]$. Malaria interventional approaches targeting the vector and which involve the use of insecticidetreated nets (ITNs), indoor residual spraying (IRS), and in some specific settings, larval control are a critical component of the multipronged attack on malaria [35]. Protection by ITNs and IRS has demonstrated a greater impact on reducing malaria [73]. However, strains of Anopheles mosquitoes developed resistance to dichlorodiphenyltrichloroethane (DDT), pyrethroid, and other insecticides, and the environmental impact of DDT was recognized [73]. Also, the Plasmodium parasites became resistant to chloroquine, the mainstay of antimalarial drug treatment in humans $[28,38]$. Due to the resistances developed by the malaria pathogens and vectors to chemicals used, and environmental concerns, there was a need for additional novel approaches in malaria prevention, control, and treatment.

\section{Acoustic prevention and control of mosquitoes}

For many years sound had been used to scare off pest species, with its humble origins of loud claps and yells in ancient agricultural fields, and now ultrasound producing electronic repellents (EMR) is used in combating mosquitoes [1, 2, 3, 27, 37, 41, 49, 59, 62, 69]. Sonic sources of different dimensions and power levels have been tested in trapping studies, population surveys, and behavioral manipulation bioassays, including general-purpose loudspeakers, large piezoplastic sheets on foam boards, acoustic lasers, small tweeters and even tuning forks [54]. Recent studies have shown a pronounced negative phonotaxis in swarming male mosquitoes (Culicidae) evoked by the audible acoustic range of 140-200 Hz [49]. The electronic mosquitorepelling devices whose results have been reported generated sounds from $2 \mathrm{kHz}$ to $80 \mathrm{kHz}$ in frequency with harmonic peaks from $4 \mathrm{kHz}$ to $68 \mathrm{kHz}[1,41]$. Experimental results on repellency testing using the electronic piezo buzzer mosquito repellent showed significant repellency in mosquitoes by the $40-55 \mathrm{kHz}$ frequency range, which was a wider bandwidth compared to the frequency range of 38-44 kHz earlier reported [41, 59]. Also, a study involving design and testing of an electronic-based pest repellent established that $20-30 \mathrm{kHz}$ of simulated ultrasonic frequency and $21-29 \mathrm{kHz}$ of experimental ultrasonic range showed negative phonotaxis in mosquitoes with the simulation and experimental results being consistent within a measuring uncertainty of $\pm 5 \%$ [1]. Also, experiments with functioning electronic mosquito repellents (EMR) mimicking calls from bats and male A. gambiae in the frequency range of $125 \mathrm{~Hz}$ to $74.6 \mathrm{kHz}$ showed that 12 out of 15 field experiments yielded higher landing rate on the human bare body parts than the control experiments $[4,15$, 27]. It was reported that EMRs that were used in indoors and outdoors repelled mosquitoes within a range

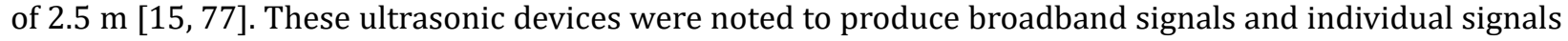
at high and low frequencies, spanning a bandwidth of approximately $43.0 \mathrm{kHz}$ [83]. Also, natural ultrasonic bat-cry signals have been observed to disrupt the behavior of night-flying insects preyed on by bats [54].

Experiments with air conditioner (AC) with an inbuilt ultrasonic device (AC-UD) under the "fan ON with ultrasonic ON", "fan ON with ultrasonic OFF", and "fan OFF with ultrasonic ON" yielded final mortality at 24 hours of $60.7 \%, 15.3 \%$ and $17.3 \%$ in the knockdown tests [62]. Evaluation experiments with mosquitorepelling devices which included the Anti-Pic $₫$, Mosquito Repeller $®$ DX-600 and Bye-Bye Mosquito ${ }$ done by exposing human hands to Aedes albopictus (Skuse) adults showed insignificant success in repellency, failing to confirm the $30.3 \%$ repellency due to Anti-Pic $®$ initially determined $[4,66]$. Based on these research findings that involved the use of natural and synthetic ultrasound have shown that ultrasound was an effective tool in the control of mosquitoes, the malaria vector through knocking down and expelling or repelling [1, 37, 41, 59, 62]. Recorded natural sounds of $O$. tormota (previously known as Amolops tormotus) and $C$. afra evoked negative phonotaxis in the mated female A. gambiae for fear of predation and neural stress [41,55]. Evasive responses characterized by $58.5^{\circ}$ antenna erection, physical injury, fatigue and falls, jammed mosquitoes' own ultrasound frequency besides immobilizing them which was attributed to pronounced stress on the nervous system and fear of predation have been noted [55, 59]. Other studies have established that mosquitoes detect ultrasound in the range of $38-44 \mathrm{kHz}$, regardless of the source, initiating avoidance response since it creates stress on their nervous system, jams mosquitoes' own ultrasound frequency besides immobilizing them 
$[55,59]$. Such evasive responses have been reported for both $10-34 \mathrm{kHz}$ and $35-60 \mathrm{kHz}$ sounds of 0 . tormota based on physical behavioral activity [55]. An average percentage startle of $34.12 \%$ and $46 \%$ was observed in mosquitoes in the $10-34 \mathrm{kHz}$ and $35-60 \mathrm{kHz}$ sounds of $O$. tormota respectively based on observable behavioural startle response [55]. Recent researches determined the Protection Index (PI) or Repellency expressed as a percent based on mosquito landing, probing, and feeding using equation 1 [4, 20,67]:

$$
\boldsymbol{P}=\frac{U P H-P}{U P H} \times 100 \%
$$

where $\boldsymbol{P H}$ is the number of mosquito bites (or initiated bites) on the supposedly protected blood meal (treatment) (obtained from an already-existing collection in Entomology Department, Kenya Medical Research Institute (KEMRI) Kisumu, Kenya), and $\boldsymbol{U P H}$ is the same measure for a supposedly unprotected blood meal (control).

The startle response in mosquitoes evoked by the natural sound of the male A. gambiae and Delphinapterus leucas had not been reported. The mimicked sounds of the male A. gambiae and the recorded natural sound $O$. tormota had been studied but also required further research due to their efficacy hence the basis for this study $[55,59]$.

\section{Mosquito biology, Audition, Mosquito mating behavior, and Electronic mosquito repellent devices}

The egg, larva, and pupa stages in the lifecycle of the A. gambiae are aquatic and last 5-14 days, depending on the species and the ambient temperature [18]. It is important to understand the lifecycle of the mosquito for effective malaria vector control. Biting female mosquitoes not only irritates people and animals but also transmit malaria [74]. The body parts of the adult stage of the mosquitoes, mainly the antennae serve an important role in communication $[59,68]$. The egg, larva, and pupa stages in the lifecycle of the A. gambiae are aquatic and last 5-14 days, depending on the species and the ambient temperature $[16,18]$. Both male and female adult mosquitoes feed on plant nectar, but the female feed on vertebrates' blood, for nutrients required for egg maturation [10,11,36,56]. The female Anopheles mosquitoes lay eggs on the surface of the water at night and under favorable conditions, hatching occurs within one or two days and develops within the aquatic habitat $[23,61]$. The A. gambiae larvae develop in permanent man-made structures and natural pools [47]. The adult mosquitoes have slender bodies consisting of the head, thorax, and abdomen; the head specialized for acquiring sensory information and for feeding [50]. The mosquito antennae also detect host and breeding sites odors [16]. The head also has an elongated, forward-projecting proboscis used for feeding and two sensory palps. The adult stages of many mosquito species are feeders of blood, which has given some disease-causing organisms a reliable mode of transmission to animal hosts. During the adult stage of the males and females Anopheles rest with their abdomens sticking up in the air and the female Anopheles mosquito act as a malaria vector [22]. The adult females Anopheles mosquitoes can live up to a month or more in captivity but they don't live more than 1-2 weeks in nature $[17,56]$.

Warm-blooded hosts provide mosquitoes with thermal contrast that facilitates the localization of a suitable blood meal $[25,53]$. An analysis of how the mosquito actually bites, probes for the blood vessels and finally sucks blood showed that the mean time taken before the mosquito starts probing after landing was 6.5 seconds, the mean probing time was 142 seconds, mean feeding time was 240 seconds (feeding times were between 150 and 329 seconds) giving a total of 389 seconds (6.5 minutes) [19]. Female Anopheles mosquitoes lay eggs on the surface of the water at night and under favorable conditions, hatching occurs within one or two days and develops within the aquatic habitat $[23,61]$. The A. gambiae larvae develop in permanent man-made structures and natural pools [47]. The adult mosquitoes have slender bodies consisting of the head, thorax, and abdomen; the head specialized for acquiring sensory information and for feeding. The mosquito antennae detect host 
and breeding sites odors [50]. The head also has an elongated, forward-projecting proboscis used for feeding and two sensory palps. The adult stages of many mosquito species are feeders of blood, which has given some disease-causing organisms a reliable mode of transmission to animal hosts. Adult males and females Anopheles rest with their abdomens sticking up in the air. It is during the adult stage that the female Anopheles mosquito acts as a malaria vector [22]. The adult females can live up to a month or more in captivity but they don't live more than 1-2 weeks in nature $[17,56]$. The mosquito has a pair of large, wraparound eyes, and a pair of long, hairy antennae; its ears projecting from the front of its face [40]. The antenna detects the particle velocity component of a sound field, which is restricted to the immediate vicinity of the sound source in the acoustic near field. Male mosquitoes require about 24 hours before their terminalia get rotated and their fibrillae mature enough to become erect and detect females whereas the female mosquitoes need 48-72 hours before they become receptive to males prior to blood-feeding in the wild [22,63]. Anopheles males can mate several times, but females become refractory to re-insemination and re-mating is rare [59]. The male Anopheles mosquitoes aggregate before dusk and initiate swarming at the onset of sunset and mating occurs during the early evening, primarily in swarms, a typical time for the mated female mosquitoes seek blood meal through bites of human beings. [12, 24, 42]. Flying mated female A. gambiae mosquitoes produce familiar $150-500 \mathrm{~Hz}$ whining sound recording maximum intensity at $380 \mathrm{~Hz}$ when searching for proteins [32, 57, 70]. Also, the auditory system of the male A. gambiae is selectively tuned to the female A. gambiae in the approximate frequency range of $300-400 \mathrm{~Hz}$ with a maximum intensity frequency being equal to that of the female A. gambiae [57,65, 70]. Ultrasound generated artificially or naturally is detected by mosquitoes evoking evasive response [59]. It has been reported that insects responded to the $2-100 \mathrm{kHz}$ sound with the sound in the $10-100 \mathrm{kHz}$ frequency range causing the unpleasant feeling due to intense auditory stress they move away from the device [41, 75]. Electronic mosquito repellents (EMRs) are designed to repel female mosquitoes by emitting high-pitched sounds $[7,27,41,77]$. Manufacturers of these electronic mosquito repellents on the market have put-forth and argument of their effectiveness in repelling or attracting mosquitoes, though not common in Africa [45]. The Anti-Pic $®$, Mosquito Repeller $®$ DX-600, and Bye-Bye Mosquito $®$ electronic mosquito repellents have been studied in order to establish their effectiveness in mosquito repellency but none has supported the claims of their $30.3 \%$ effectiveness in mosquito repellency [4]. Additionally, Electronic Mosquito Repellents (EMRs) in the market have yielded low mosquito repellency of between $20.0-30.3 \%$. The low repellency rates of the EMRs ("repellent radio") could be due to the narrow bandwidth size of $15 \mathrm{kHz}$ [4, 45]. It was also noted that in 12 of the 15 experiments, the landing rates of mosquitoes on the human participants in the groups with functioning EMR were actually higher than in the control groups [27]. A designed electronic ultrasonic device that swept sound waves in the range of $20-70 \mathrm{kHz}$ yielded mosquito repellency in the $40-55 \mathrm{kHz}$ frequencies due to stress on the nervous system of mosquitoes [41]. Based on the experimental data on average mosquito bites, the protection index evoked by the $40-55 \mathrm{kHz}$ sound was determined using equation 1 yielding a protection index of $68.99 \%$ [41]. Additionally, an electronic-based pest repellent device designed to produce sound in the frequency range of up to $80 \mathrm{kHz}$ with the $20-30 \mathrm{kHz}$ and $21-29 \mathrm{kHz}$ ranges due to simulation and experimental ultrasound respectively was noted to repel mosquitoes and bugs [1]. Ultrasound causes nervous stress to the mosquito and at the same time evokes fear due to predation or/and further mating depending on the source of the natural ultrasound $[1,59]$. The studies about the repellency of the female A. gambiae using the hearing mechanism was still a viable venture based on the confirmation that in both males and females, the antennae are resonantly tuned mechanical systems that move as simple forced damped harmonic oscillators when acoustically stimulated, despite the differences in the hearing ability [49].

\section{The natural ultrasound generators: Odorrana tormota, and Delphinapterus leucas}

The Odorrana tormota species is a frog restricted to Huangshan in Anhui Province, and Jiande and Anji counties in Zhejiang Province, China and generates ultrasounds through vocal apparati and uses the frequency range of up to $128 \mathrm{kHz}$ for communication [48, 71, 72]. During the reproductive season, males emit a variety of highpitched calls at night with energy spectrums extending into the ultrasonic range [71]. Recent research with the 
O. tormota calls showed some degree of downward frequency modulation with a subset of calls having a carrier of constant frequency $(5,72)$. As observed in recent studies, ultrasound from 0 . tormota can play a critical role in malaria vector control by evoking evasive responses in malaria vectors since their call frequencies stretch beyond the determined startling frequency range of $20-30 \mathrm{kHz}, 21-29 \mathrm{kHz}, 38-44 \mathrm{kHz}$, and $40-55$ $\mathrm{kHz}$ in mosquitoes $[1,41,55,59]$. The sound of 0 . tormota having exhibited the greatest startle responses in mosquitoes in recent findings provided a grounding for further investigation in its startle effect on the female $A$. gambiae besides exploring other natural sounds of the male A. gambiae, and $D$. leucas. The beluga whale, $D$. leucas which also generates sound naturally is a medium-sized toothed whale, which becomes completely white when it reaches sexual maturity around seven years of age [21,39]. Adult male beluga whale, D. leucas attain a length of 4.5 meters and females 3.5 meters and are similar in appearance [21]. Young ones are born dark grey and gradually become paler as they mature spending the summer in coastal and offshore areas [21]. Their distribution of the beluga whale, $D$. leucas is centered on certain river estuaries, which they visit shortly after ice break-up and where they molt $[21,26,51]$. The beluga whale, $D$. leucas have a mean lifespan of between 15 to 30 years though they may live beyond 40 years $[21,39]$. The beluga whale, $D$. leucas are sexually mature at the ages of 5-7 years and adults are capable of giving birth every 3 years. The beluga whales feed on a variety of fish and invertebrates. The polar bears and the Inuit hunters are the main predators of whales [21; 26]. Cetaceans produce frequency-modulated sounds and amplitude-modulated sounds with $D$. leucas producing signals with peak frequencies of 40 to $60 \mathrm{kHz}$ in San Diego Bay, California, and 100 to $120 \mathrm{kHz}$ in Kaneohe Bay, Hawaii [13]. The "nonlinear phenomena" spectral features discovered in marine mammal vocalizations include frequency jumps, subharmonics, biphonation, and deterministic chaos [30;48]. The 0 . tormota and $D$. leucas use sounds for echolocation and communication purposes [58,64]. The call consists of several harmonic segments (Ha) having multiple harmonics with energy extending into the ultrasonic range, a signal break $(\mathrm{Br})$, as well as two of the nonlinear characteristics which include chaos (Ch) and subharmonics (Sh) [29].

\section{Statement of the Problem}

Africa and the world as a whole suffer both economic and health burden due to malaria. Interventions which include the use of chemicals targeting malaria pathogens and vectors have led to a decline in malaria mortality and morbidity though at a slower rate due to buildup of resistance. Also, the use of electronic mosquito repellents mimicking the sounds of bats or male mosquitoes in the control of mosquitoes has been a debatable issue since the $30.3 \%$ claimed repellency failed to be confirmed. However, studies on mosquito startles involving the use of the recorded sound of 0 . tormota have been carried out yielding an average startle of $34.12 \%$ and $46 \%$ in mosquitoes in the $10-34 \mathrm{kHz}$ and $35-60 \mathrm{kHz}$ frequency bands. These startle values were determined from initial physical behavioral responses without an attractant. Additionally, the protection index evoked by the 40$55 \mathrm{kHz}$ sound band against mosquito bites was $68.99 \%$. Improved experimental setups involving the use of air conditioner (AC) with an inbuilt ultrasonic device (AC-UD) under the "fan ON with ultrasonic ON", and "fan OFF with ultrasonic ON" gave 24-hour mortality of $60.7 \%$, and $17.3 \%$ in mosquitoes. Therefore there was a need to use an improved bioassay setup to and natural sound sources in order to determine and analyze the landing rates and behavioral startle responses of the mated female A. gambiae on warm bloodmeal attractant evoked by the individual sound of the male mosquito, A. gambiae, male 0 . tormotas, and male and female D. leucas. The data collected was based on the number of mosquitoes that approached the attractant; landed; landed and probed; or landed, probed and bit or fed the repellent-treated blood meal in the treatment bioassay chambers and the untreated meal in the Control chamber. The protection index (PI) against mosquitoes due to the animal sounds was determined and compared. The results determined confirmed the feasibility of using recorded animal sound as an additional malaria intervention measure.

\section{The bioassay}

The biological assays (bioassays) are experiments that use living organisms, with repellent bioassays 
involving mosquitoes. There are three biological assay procedures for repellents which include ASTM E951 (laboratory testing of non-commercial repellent formulations on the skin), ASTM E939-94 (field testing topical applications of compounds as repellents for medically important and pest arthropods and mosquitoes), WHO/ Control of Tropical Disease/WHO Pesticide Evaluation Scheme/Informal Consultation WHO/CTD/WHOPES/ IC/96.1 (Report of WHOPES informal consultation on the evaluation and testing of insecticides) and the U.S. Environmental Protection Agency (EPA) Office of Prevention, Pesticides, and Toxic Substances, USEPA OPPTS 810.3700 (product performance test guidelines) [9, 13]. A stimulus is applied and the response observed repeatedly for a population and the response estimated with the desired level of precision [46]. In repellent bioassays, the stimulus is normally a dosage of repellent applied to human skin, to the skin of an animal subject, or to an inanimate object such as fabric, membrane, or filter paper [8]. Recent researches on repellency of mosquitoes involved the use of a Y-tube olfactometer setup for behavioral assay shown in Fig 1 as a bioassay cage, a design modified and adopted for this study [66].

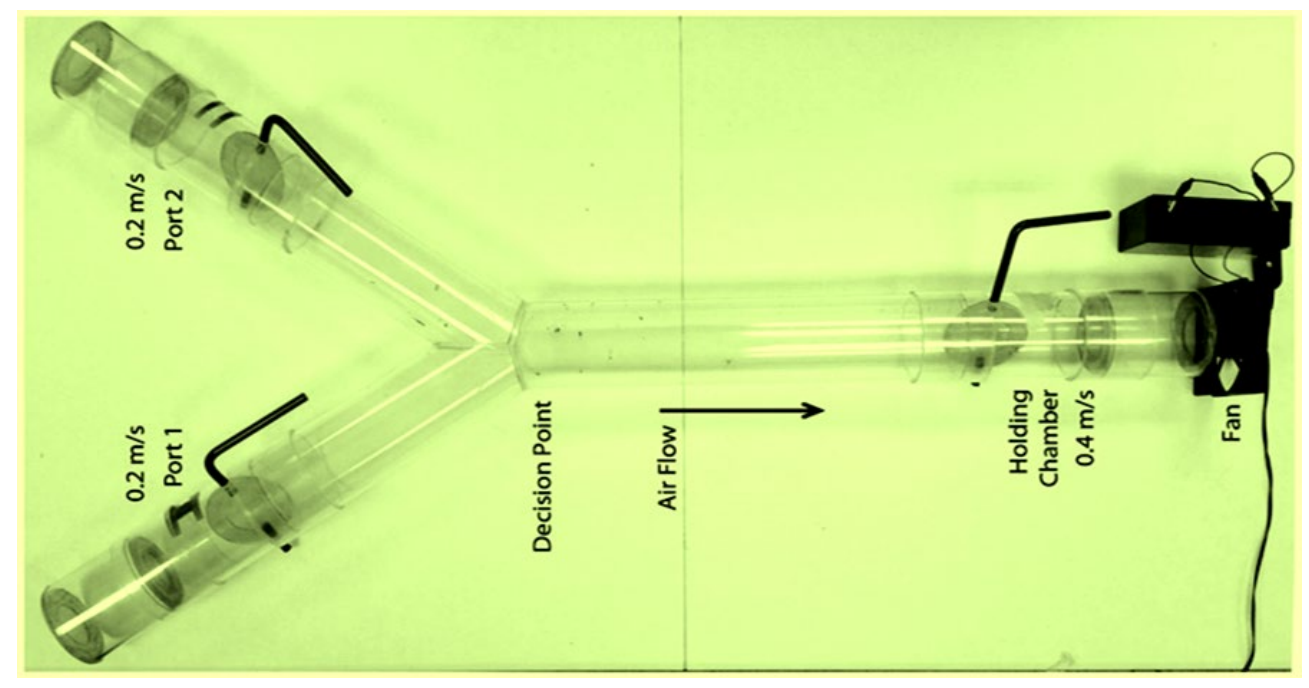

Fig 1: The Y-tube used in the attraction-inhibition assays

Source: [66]

This study preferred the use of cow (Bos tarsus) whole blood meal as a food attractant to the mated female $A$. gambiae s.s. The mated female A. gambiae s.s. had shown a preference of cow blood compared to blood from other animals with a significantly higher probability of laying eggs after feeding on human and cow blood, compared to chicken or dog blood meal $[43 ; 52 ; 60]$. Mosquitoes pick up cues that indicate the presence of animals or humans through the vision for spotting the host and thermal sensory information to detect body heat $[25,53]$.

\section{Objectives}

\section{General Objective}

Investigate the startle response of the African female Anopheles gambiae by the natural sound of male Anopheles gambiae, male Odorrana tormota, and male and female Delphinapterus leucas.

\section{Specific Objectives}

(i). Determine the number of the mated female A.gambiae s. $s$ approaching, landing, and probing the blood meal evoked by the individual sound of the male mosquito, A. gambiae, male $O$. tormota, and male and 
female D. leucas.

(ii). Investigate the acoustic behavioral startle responses of the mated female A. gambiae s. $s$ on blood meal evoked by the individual sound of the male mosquito, A. gambiae, male 0 . tormota, and male and female D. leucas.

(iii). Evaluate the protection index (PI) against the female A. gambiae due to the sounds of male mosquito, $A$. gambiae, male $O$. tormota, and male and female D. leucas.

\section{MATERIALS AND METHODS}

\section{The study area}

The research conducted in Kenya Medical Research Institute (KEMRI)/Centers for Disease Control and Prevention (CDC) entomology laboratories, Kisumu, Kenya involved the rearing of both male and female mosquitoes, A. gambiae s. s, recording of mosquito sounds and bioassay studies. The sounds of the Chinese frog, O. tormota recorded from Huangshan Hot Springs in Anhui Province, China were acquired from Prof. Albert Feng of the University of Illinois at Urban-Champaign. Also, the sounds from the $D$. leucas were acquired from Prof. Herve Glotin of Institut Universitaire de France [33; 34].

\section{Study animals and rearing conditions}

The male O. tormota, male and female A. gambiae s.s, D. leucas, and T. truncatus were used in the study. Mosquito rearing and feeding was guided by Standard Operation Procedures for Anopheline mosquito rearing and maintenance, SOP No. 3005/ENT/014 for KEMRI. The male and female A. gambiae s. s mosquitoes were bred and reared at KEMRI/CDC entomology laboratories under $80 \pm 10 \%$ Relative humidity (RH), $27 \pm 2{ }^{\circ} \mathrm{C}$ temperature, and equal light-darkness hour cycle with one-hour dawn dimming as outlined in SOP No. 3005/ ENT/014. Larvae were reared on larval pans which were filled with rainwater to a depth $1.0-2.0 \mathrm{~cm}$ and the room temperature was maintained at $30 \pm 2{ }^{\circ} \mathrm{C}$ as given under SOP No. 3005/ENT/014. The larvae were fed on a combination of Tetramin baby fish food and Koi's choice premium fish food in the ratio of 1:2 with the quantity of food and feeding frequency determined by the stage, size, and density of larva. The pupae of the A. gambiae s. s which do not feed were reared in covered glass vials quarter filled with rainwater at $28{ }^{\circ} \mathrm{C}$ under standard laboratory conditions. Both male and female A. gambiae s.s were fed on a $10 \%$ sugar solution, though the female A. gambiae s. s were additionally fed on blood meal 3-7 days post-emergence. The female mosquitoes, $A$. gambiae s.s were separated from the male A. gambiae s.s from a swam of mosquitoes based on their mouthparts and affinity to a blood meal. Twenty-five sets, each of fifty; 3-5 day old mated female A. gambiae s. s obtained from emerged mosquitoes were reared separately at KEMRI/CDC Entomology laboratories under controlled conditions. Also, a set of one hundred male A. gambiae s.s were reared separately under similar conditions. The bioassays which were sound-based were conducted in a quiet and well-lit room in KEMRI/CDC entomology laboratory under controlled room conditions.

\section{Sound recording and playback equipment}

A computer running on the Windows operating system and office with the mounted sound card was used in the study. The computer was installed with the Avisoft-SAS LAB Pro version 5.2 software for sound recording and playback. The computer was mounted with the hardlock key that enabled the running of the Avisoft-SAS LAB Pro version 5.2 program. The input and output ports on the computer served as inputs for the signal from the Avisoft recorder. The recorder consisted of the Avisoft UltraSoundGate (model 112) and running on the RECORDER USG (rec_usg.exe) software. The sound was played through a vifa external ultrasonic speaker with frequency range $( \pm 12 \mathrm{~dB}): 1-120 \mathrm{kHz}$, impedance: $4 \Omega$ and sensitivity at $50 \mathrm{kHz}: 92 \mathrm{~dB} / 2.83 \mathrm{~V} / 1 \mathrm{~m}$. 


\section{Recording and filtering of the animal sounds}

A set of 100 male A. gambiae s.s obtained the reared 3-5 days old mosquitoes were transferred into a cylindrical glass cage covered at both ends with netting by means of an aspirator as given in Fig 2 . The sounds of the male and mated female A. gambiae s.s were recorded separately using the Avisoft recorder at a sampling frequency of $500 \mathrm{kHz}$ at 16 bit and saved as a .wav file in the hard disc. The omnidirectional microphone, set to default and connected to the AvisoftUltraSoundGate (model 112), was connected to the computer through the universal serial bus (USB) port. The Avisoft-SAS LAB Pro, version 5.2 software was initiated and the microphone directed to the source of the sound. With the gain on the AvisoftUltraSoundGate (model 112) adjusted to an appropriate level to avoid overmodulation and the recording level from the computer set to $20 \mathrm{~dB}$, the recording button was pressed to record the sound (Manga're et al, 2012).

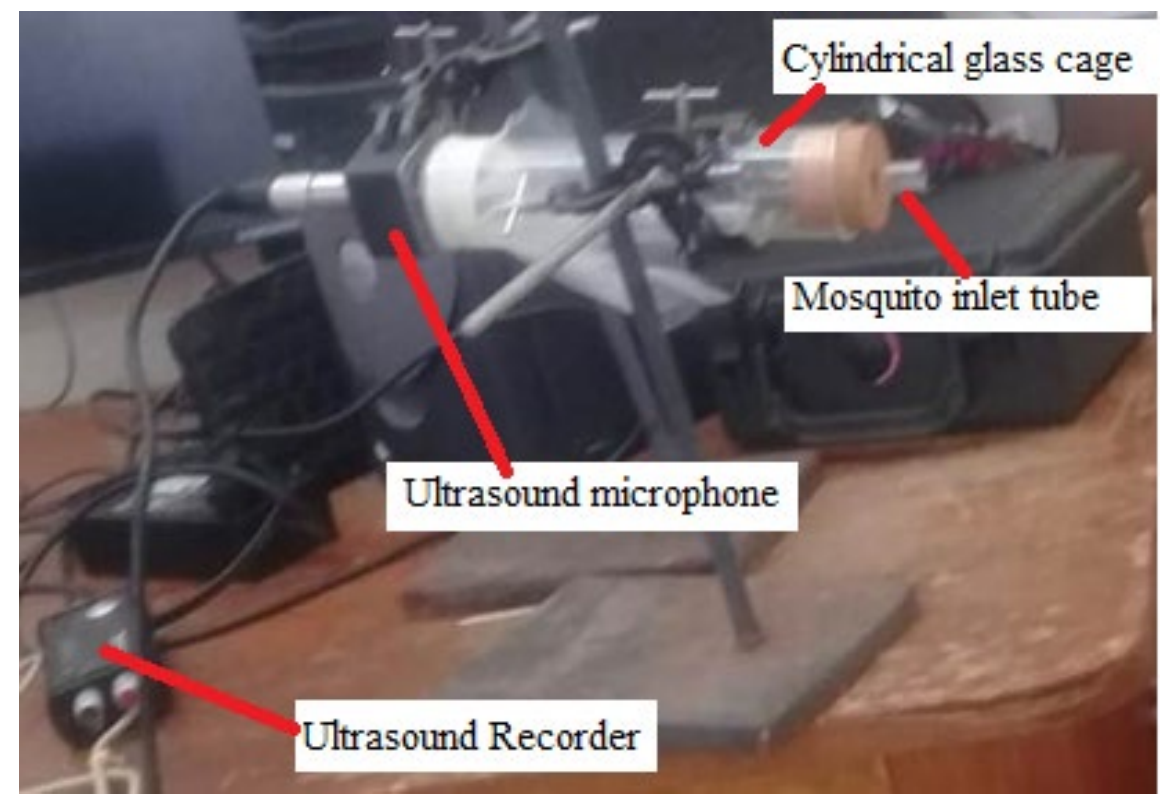

Fig 2: The sound recording setup

The following settings were made to the Avisoft software for recording and analysis purposes: the time domain filter (Finite Impulse Response - FIR) option the filter type the upper cut-off frequency, $\mathrm{f}_{\text {uco }}=140 \mathrm{kHz}$ and $\mathrm{f}_{\mathrm{lco}}=$ $0 \mathrm{kHz}$ for the male A. gambiae. From the tools option, the calibration was set to sound pressure level (SPL) with reference to sound and the SPL reference was $20 \mu \mathrm{Pa}$. The Fast Fourier transform (FFT), an option under the spectrogram parameters was set to 512 and hamming window selected for the display. Also, the temporal resolution overlap was set to $50 \%$ with the color palette set to graypal. The frame size was set to $100 \%$ for realtime spectrogram parameters and the black and white box $(\mathrm{B} / \mathrm{W})$ checked for display. Besides, the envelope was also set to the original waveform whereas the pulse detection was set to gate function. The same settings were replicated in the Raven Pro. 1.4 software. The recorded sounds of the male mosquitoes were saved in the hard disc as malemosquitosound.wav.

The clips of recorded sound of male 0 . tormota recorded by 702 digital recorders from the Huangshan Hot Springs, Anhui Province in China at a sampling frequency of $192 \mathrm{kHz}$ were acquired through Prof. Albert Feng, Illinois University. The sounds of the mixed male and female D. leucas recorded using the Wavshark system, C75, and the $\mathrm{C} 55$ hydrophone at a sampling frequency of $128 \mathrm{kHz}$ when they were swimming in the tank of the Vanaqua were acquired from Prof. Herve Glotin of Institut Universitaire de France [33]. The sounds of male A. gambiae, male 0 . tormota, and mixed male and female $D$. leucas were further subjected to band-pass filters 
incorporated in both the Avisoft and Raven Pro software, yielding the 10-34 kHz frequency band informed by the acoustic repellency bands evoking negative phonotaxis $[1,37,41,59,62]$.

\section{The bioassay}

Feeding and maintenance of both male and female A. gambiae s. $s$ was conducted as outlined in the Standard Operating Procedures, SOP No. 3005/ENT/014. The cow blood meal which is preferred by the A. gambiae s.s was obtained from a slaughterhouse, processed and stored in Kenya medical research institute as per the Kenya Medical Research Institute (KEMRI)/ Centre For Global Health Research (CGHR) Standard Operating Procedures (SOP) for collecting blood for blood-feeding insects in the laboratory and SOP No. 3005/ENT/014. Active 3-5 day old mated female A. gambiae s.s which had been starved for 24 hours and of high affinity to the blood meal were selected for the bioassay. Recorded natural sounds of the male 0 . tormota, male A. gambiae, and the sound of male and female $D$. leucas were used in the bioassay as the treatment bioassay. The repellency of $46 \%$ elicited by the $35-60 \mathrm{kHz}$ recorded sound of 0 . tormota in mated female A. gambiae s. $s$ in recent research findings prompted a further study in the natural and synthetic sounds of the male 0 . tormota. The individual natural and sounds of male $O$. tormota, male A. gambiae s. $s$ and D. leucas were filtered into $10-34 \mathrm{kHz}$ frequency bands using filters in the Avisoft-SAS LAB Pro version 5.2. The 10-34 kHz sounds were broadcasted into the one of the bioassay chambers as a treatment to the blood meal and behavioral responses of the mated female A. gambiae s. $s$ observed and recorded. A $1.0 \mathrm{~m}$ long modified standard Y cage called fighto-Y bioassay glass cage fitted with a mosquito netting on the three cross-section areas A, B, and C shown in Fig 3 was used in the bioassay [66]. Cotton wool was used to seal the entry/ exit hole on the net placed on face $C$ of the fighto-Y glass cage. The cage was divided into three sections, A, B, and the Neutral chamber (C). The open ends of chamber A, $B$, and $C$ were covered with a mosquito netting with net $A$ and $B$ were in contact with the cellulose membrane covering the warm blood contained in the feeding chamber. The feeding chamber connected to the Hemotek membrane feeding apparatus was used to feed the blood-sucking mated female A. gambiae s. $s$ through an artificial membrane as described in the SOP No. 3005/ENT/014. The blood chamber which was an aluminium cylindrical container was loaded with fresh blood by means of a Pasteur pipette through the ports at its back. The ports were covered with a removable rubber material. The loaded blood in the chamber was covered with an artificial cellulose membrane and connected to the netting on face A and B as given in Figs 5 and 6 . The cellulose membrane, attached to the netting, allowed for mosquito landing, probing (bites), and sucking of the blood meal (cow) which was maintained at the body temperature of a healthy cow of $38.60^{\circ} \mathrm{C}$ by the Hemotek membrane feeding apparatus as given in Fig 4 [44]. The duration of the bioassay study was measured using a digital timer.

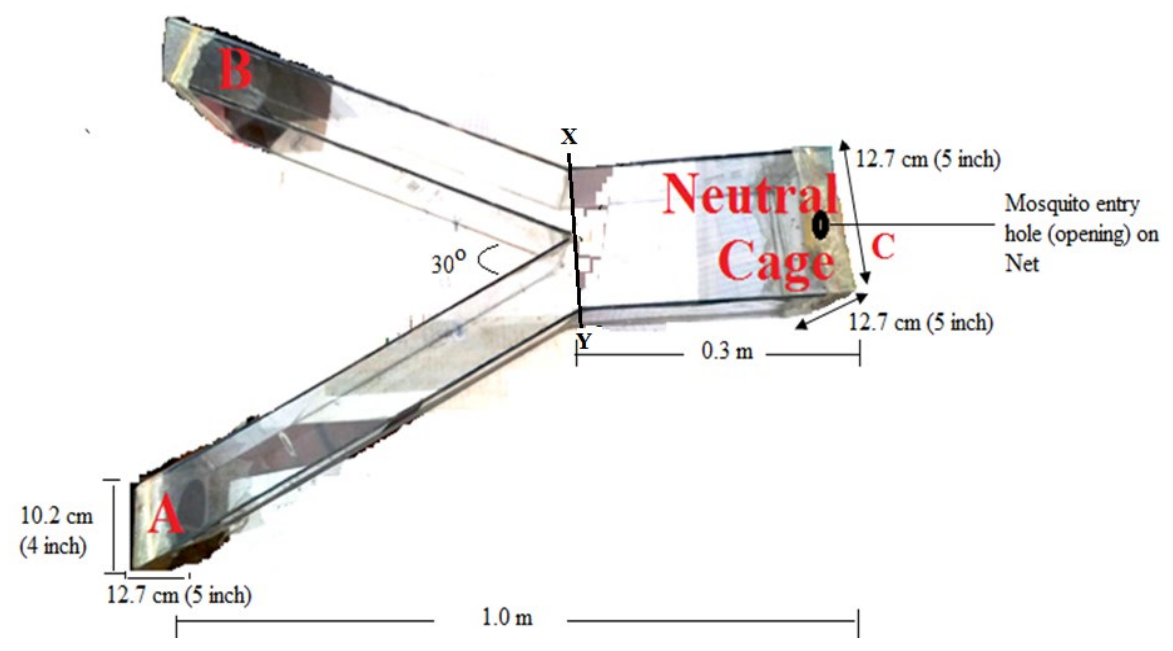

Fig 3: The fighto-Y glass cage for the repellency bioassay 


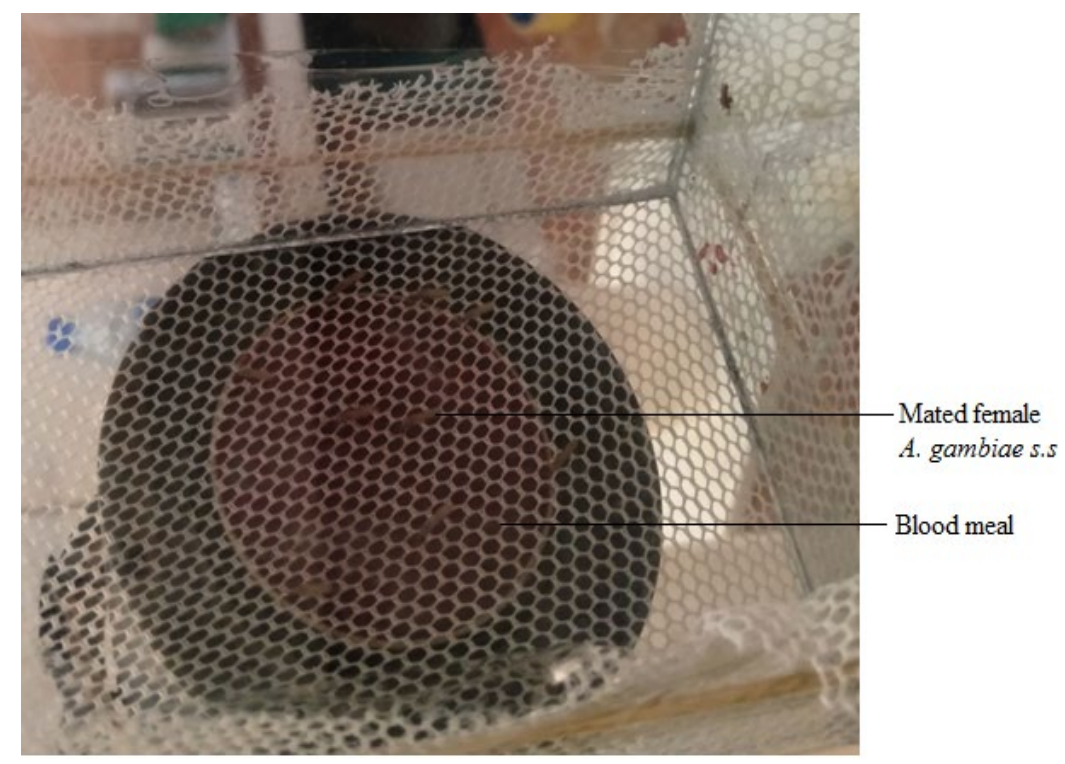

Fig 4: Bloodmeal in the chamber mounted on the net by means of retort stand

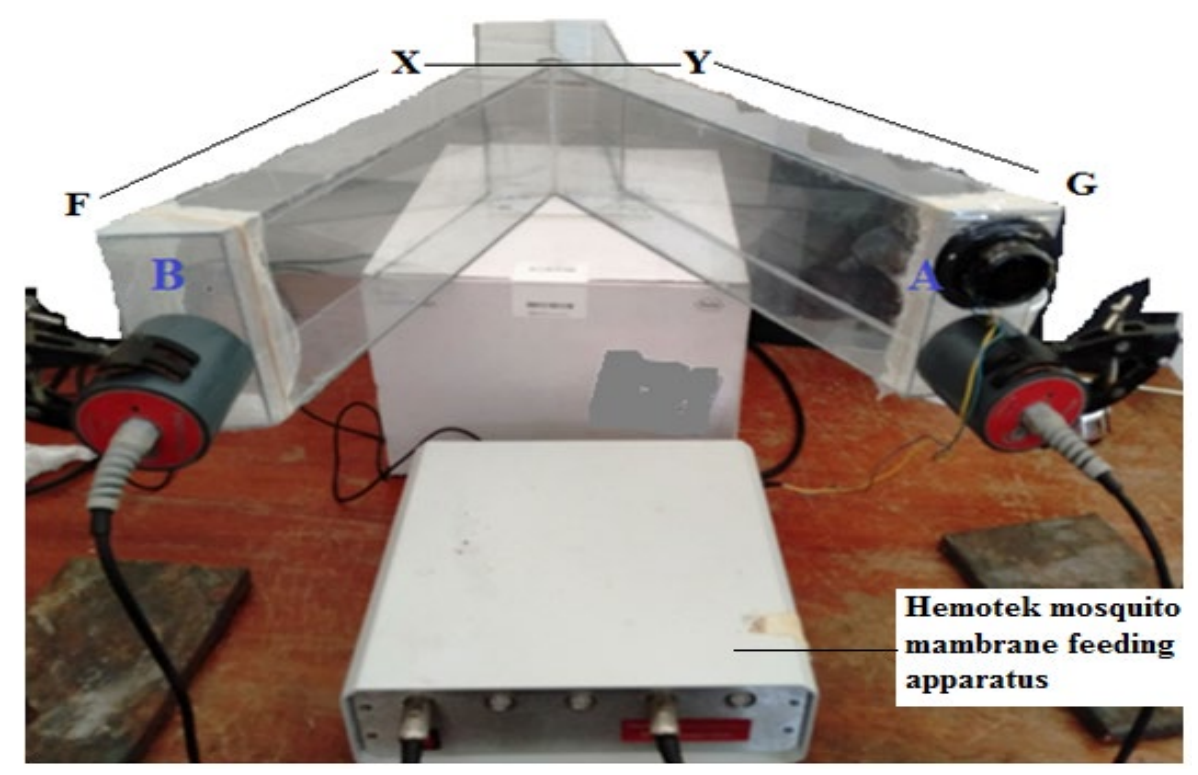

Fig 5: Bioassay set-up with A as treatment chamber 


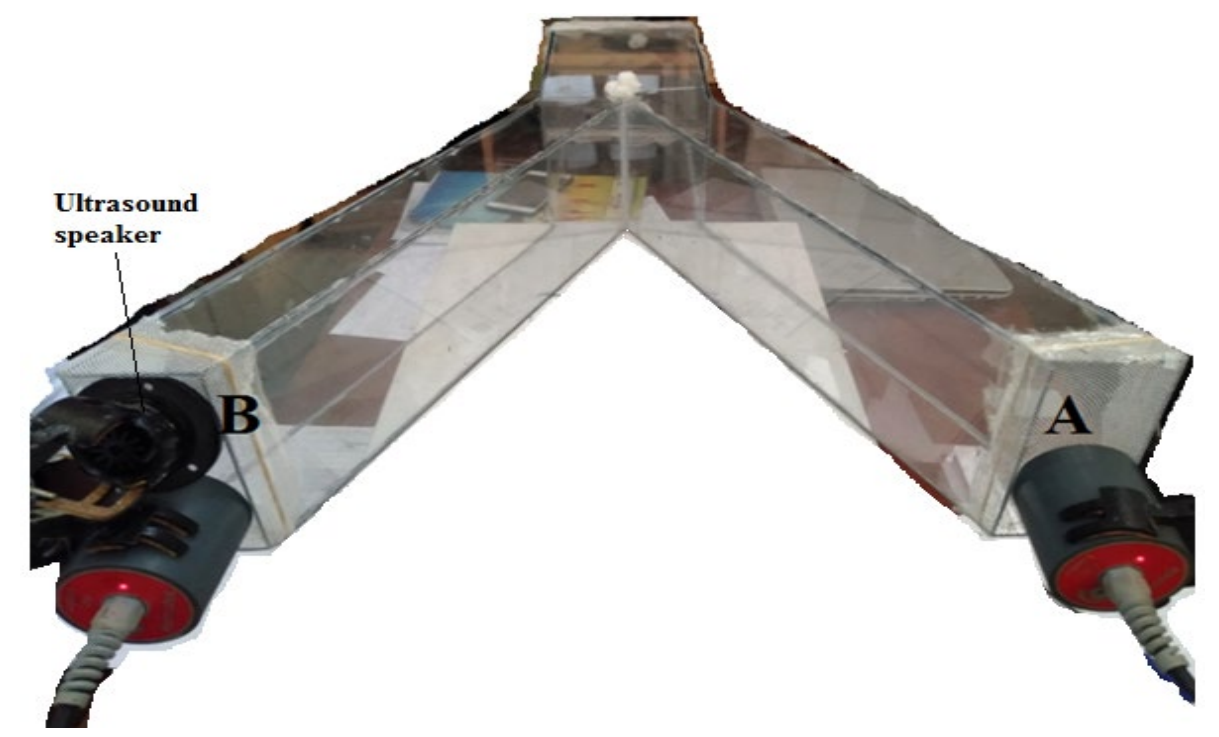

Fig 6: The bioassay set up with treatment on chamber $B$

(i). Determination of the number of the mated female A. gambiae s. $s$ approaching, landing, and probing the blood meal evoked by the individual sound of the male mosquito, A. gambiae, male 0 . tormota, and male and female $D$. leucas.

The $10-34 \mathrm{kHz}$ natural sounds of the male 0 . tormota, male A. gambiae, and mixed male and female D. leucas which were the treatment in our bioassay study were allowed through the netting on side A and B of the fighto-Y cage interchangeably as given in Fig 5 and 6 to avoid bias. The blood meal in the treatment chamber was placed $2.0-3.0 \mathrm{~cm}$ from the source of the $10-34 \mathrm{kHz}$ sounds of the male 0 . tormota, male A. gambiae, and male and female D. leucas [6]. Two sets of bioassays, the treatment, and control experiments were performed simultaneously by exposing 50 mated and starved female A. gambiae to cow blood meal in a fighto $\mathrm{Y}$ cage under controlled laboratory conditions of $25 \pm 2{ }^{\circ} \mathrm{C}$ and $70 \pm 10 \%$ relative humidity. The bioassay study conducted in the chamber in which the starved and mated female A. gambiae s.s were exposed to a blood meal and no sound was the control whereas the bioassay study in the chamber in which the starved and mated female A.gambiae s.s were exposed to blood meal and sound was the treatment as given in Fig 5 and 6. The study was based on the in vitro method ("in the glass") and the ASTM E951-94 repellent procedures with the treatment being the various frequency bands of natural and synthetic animal sounds [13].

Fifty laboratory-reared mated and starved female A. gambiae were allowed into the neutral chamber by means of an aspirator through a $1.0 \mathrm{~cm}$ diameter opening on the netting at the neutral chamber of the fighto-Y glass cage. The hole was covered using a piece of cotton wool. The number of starved and mated female A. gambie s.s that occupied Chamber $\mathrm{B}$ from point $\mathrm{X}$ to $\mathrm{F}$ and the number of starved mated female A. gambie s.s occupying chamber $A$ from point $Y$ to $G$ as shown in Fig 5 were considered to have approached the blood meal in chamber $\mathrm{B}$ and A respectively. However, the number of starved mated female A. gambie s.s that remained in the neutral chamber were considered indecisive. Position XY on Fig 5 and 6, which was the decision point for starved mated female A. gambie s.s was $0.30 \mathrm{~m}$ from the point of release of the starved and mated female A. gambie s.s into the fighto-Y cage (66). The mated female A. gambiae s. $s$ had an equal likelihood in the choice of chamber A or B at point XY of the fighto-Y cage. The $10-34 \mathrm{kHz}$ natural animal sounds were played separately for a duration of $1,200 \mathrm{~s}$, and the number of female $A$. gambiae that approached the blood meal; landed on blood meal; landed and probed the blood meal in chamber A and B for a duration of $120 \mathrm{~s}$ determined and recorded [8]. 
(ii). Investigation of acoustic behavioral startle responses of the mated female A. gambiae $s$. $s$ on blood meal evoked by the individual sound of the male mosquito, A. gambiae, male O. tormota, and male and female D. leucas.

The phonotaxis behavioral parameters of the mated female A. gambiae s. $s$ evoked by natural and synthetic animal sound on exposure to a blood meal included and not limited to the level and nature of flight, activeness, nature of movement on the surface of rest, postural adjustments, steering from the ultrasound, nature of landing on the floor surface of the chamber, engorgement of the abdomen, mobility, composure, wingbeat frequency, and the hindleg extension.

Fifty 3-5 day old starved and mated female A. gambiae $s, s$ were allowed into the neutral chamber by means of an aspirator and allowed $10.0 \mathrm{~s}$ to settle and decide on either to enter the treatment chamber or the control chamber or remain in the neutral chamber voluntarily. The starved and mated female A. gambiae s. $s$ in the control chamber were only exposed to warm blood meal whereas the starved and mated female A. gambiae s. $s$ in the treatment chamber were exposed to a warmblood meal and the $10-34 \mathrm{kHz}$ filtered natural animal sounds. The starved and mated female A. gambiae s. $s$ in the neutral chamber were neither exposed to the warm blood meal nor the $10-34 \mathrm{kHz}$ filtered natural and synthetic animal sounds. The behavioral responses of the starved and mated female A. gambiae $s, s$ in the control chamber and treatment chamber were simultaneously observed as a group at an interval of $120 \mathrm{~s}$ for a duration of $1200 \mathrm{~s}$.

(iii). Evaluation of the protection index (PI) against the female $A$. gambiae due to the sounds of male mosquito, A. gambiae, male $O$. tormota, and male and female $D$. leucas.

The number of the mated female A. gambiae approaching, landing and probing the blood meal, and the behavioral startle responses of the mated female A. gambiae s. $s$ on blood meal evoked by animal sound was determined through observation and physical counting from the bioassay setup and experiments described in 2.6 and 2.7. The data on the number of the mated female A. gambiae approaching, landing and probing the blood meal, and the behavioral startle responses of the mated female A. gambiae s. $s$ on blood meal evoked by animal sound was recorded in excel worksheet of Office 2007 for means and graphical analysis. Further analysis of the data regarding the correlation and significance level essential in hypotheses testing was achieved through SPSS version 16. The behavioral startle responses of the mated female A. gambiae on food attractant evoked by the $10-34 \mathrm{kHz}$ natural sounds of male and female $D$. leucas, male $A$. gambiae, male and male $O$. tormota was determined through observation and recording of unique behaviors compared to the behavior of the mated female A. gambiae in the control chamber. The protection index was determined using equation 1 based on the number of starved and mated female A. gambiae s.s that approached the blood meal or landed and probed the blood meal in chamber A and B which was a measure of the negative phonotaxis of the starved and mated female A. gambiae s.s to sound.

\section{RESULTS AND DISCUSSION}

The 10-34 kHz acoustic propagation parameters for the sound of the male mosquito, $A$. gambiae, male 0 , tormota, and mixed male and female D. leucas.

The $10-34 \mathrm{kHz}$ filtered sounds of the male 0 . tormota, male A. gambiae, and male and female D. leucas were used in the study, and their acoustic energy and power are given in Table 1.

Table 1: The 10-34 kHz spectral acoustic power and energy

\begin{tabular}{|c|c|c|c|}
\hline & \multicolumn{3}{|c|}{$\mathbf{1 0 - 3 4} \mathbf{~ k H z}$ Sound Samples } \\
\hline Parameter & A. gambiae & D. leucas & O. tormota \\
\hline Aggregate entropy (bits) & 4.58 & 4.57 & 2.32 \\
\hline
\end{tabular}




\begin{tabular}{|c|c|c|c|}
\hline Average entropy (bits) & 4.58 & 4.57 & 2.32 \\
\hline Average power $(\mathrm{dB})$ & 51.60 & 39.30 & 71.00 \\
\hline Delta power $(\mathrm{dB})$ & 35.80 & 28.50 & 22.40 \\
\hline Minimum energy $\left(\mathrm{Pa}^{2} \mathrm{~s}\right)$ & 0.00014 & 0.0001 & 0.00025 \\
\hline Maximum energy $\left(\mathrm{Pa}^{2} \mathrm{~s}\right)$ & 0.92 & 2.29 & 6.74 \\
\hline Mean energy $\left(\mathrm{Pa}^{2} \mathrm{~s}\right)$ & 0.01 & 0.03 & 0.44 \\
\hline Maximum entropy $(\mathrm{bits})$ & 4.58 & 4.57 & 2.32 \\
\hline Maximum power $(\mathrm{dB})$ & 64.50 & 50.90 & 90.00 \\
\hline Minimum entropy $(\mathrm{bits})$ & 4.58 & 4.57 & 2.32 \\
\hline Peak power $(\mathrm{dB})$ & 64.50 & 50.90 & 90.00 \\
\hline
\end{tabular}

The filtered sound of the male A. gambiae recorded greatest aggregate entropy, average entropy, maximum entropy and minimum entropy of equal measurements of 4.58 bits correspondingly exceeding the parameters of the sound of the male 0 . tormota and mixed male and female $D$. leucas by 2.26 bits and 0.01 bits. The Average power of the sound of the male A. gambiae e was less than the average power of the sound of the male $O$. tormota by $19.40 \mathrm{~dB}$ but exceeded the respective parameters of the mixed male and female D. leucas by 12.30 $\mathrm{dB}$. The sound of the male 0 . tormota was greatest exceeding the respective parameters of the sound of the male A. gambiae and mixed male and female D. leucas by $0.43 \mathrm{~Pa}^{2} \mathrm{~s}$ and $0.41 \mathrm{~Pa}^{2}$ s. The variations in amplitude and frequency affected the change in power, with the sound of the male A. gambiae exceeding the change in power for the sound of mixed male and female $D$. leucas and male 0 . tormota by $7.30 \mathrm{~dB}$ and $13.40 \mathrm{~dB}$ respectively.

Determination and analysis of the number of the mated female A. gambiae s. $s$ approaching, landing and probing the blood meal; behavioral startle responses and protection index (PI) evoked by the sound of the male mosquito, A. gambiae, male 0 . tormota, and male and female D. leucas

The study was informed by the effect of the sound of 0 . tormota on the female A. gambiae s.s based on observable initial behavioral responses without a mosquito attractant which yielded a startle response of $34.12 \%$ showing efficacy in the use of sound in malaria vector control [55]. The current bioassay setting involved new sound clips of male $O$. tormota which required further investigation to determine the protection index (PI). A total of 50 female A. gambiae were allowed into the neutral chamber by means of an aspirator at a time and allowed to enter the open Control bioassay chamber or the treatment bioassay chamber with an equal chance of probability as shown in Fig 3 and 4.

\section{(i). Determination and analysis of the landing rates and behavioral startle responses of the mated female $A$. gambiae on food attractant evoked by the 10-34 kHz sound of the male 0 . tormota}

The control bioassay experiment was conducted in one chamber (control chamber) void of the treatment sound. In the control chamber, the female A. gambiae were observed flying freely towards the blood meal. The female $A$. gambiae flew about and rested on the glass walls and net at normal posture, projecting its abdomen in the air at about $45^{\circ}$. Low flights and landings on blood meal were observed. Fully fed female A. gambiae whose abdomen was engorged and appeared red flew lowly and rested at the base and at times on the wall with minimal movements. Some fully fed mosquitoes flew out of the control chamber and rested on the net in the neutral chamber as given in Fig 7. The low flights were attributed to the weight of the mosquito which had increased. Free flight from the neutral chamber to the control chamber and back among the mosquitoes was noted. The mosquitoes appeared relaxed. However, the bioassay chamber in which the warm blood meal was treated with the $10-34 \mathrm{kHz}$ sound of $O$. tormota showed a reduced number in the mosquitoes approaching and/ or landing on the blood meal. Restlessness and rubbing of wings and extension of hind legs were observed. The mosquitoes which had entered the treatment chamber exhibited flights along the walls, bouncing on the wall and moved towards the neutral chamber. The mosquitoes which managed to land, probe, and feed flew out of 
the treatment chamber to the neutral chamber resting on the net. Two mosquitoes were observed shaking the body while feeding while others landed on the meal and flew away without feeding. The frequency of flights in and out of the chamber increased with the fully fed mosquitoes exhibiting immobilized. This was attributed to neural stress and fear of predation.

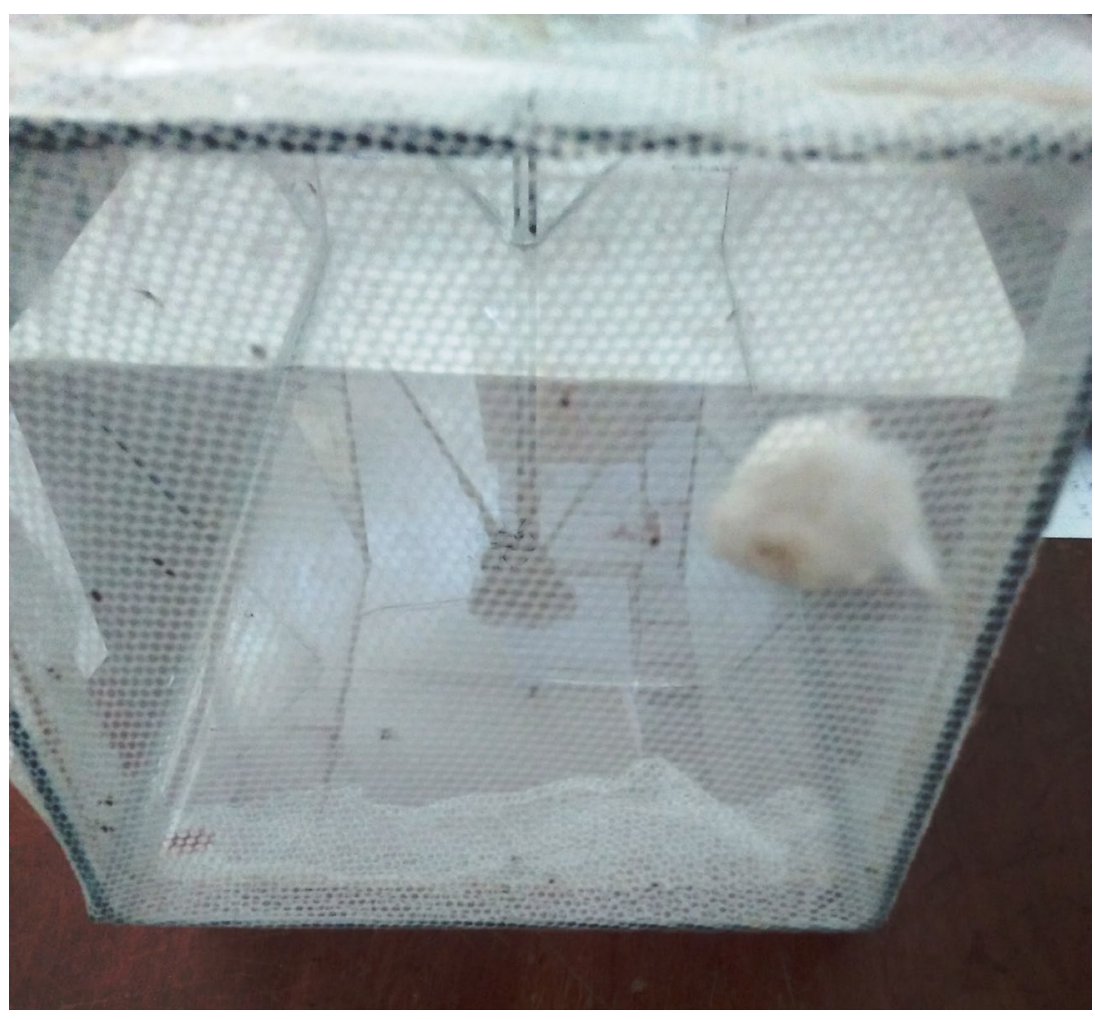

Fig 7: Mosquitoes resting on the net in the neutral chamber

All the mosquitoes in the control and treated chamber including those that landed successfully or unsuccessfully probed and fed were considered to have approached the blood meal. Based on this premise, the number of the female A. gambiae that approached the control chamber exceeded the number of the mosquitoes that approached the treatment chamber significantly $\left(\mathrm{p}=8.5381 \times 10^{-6} \ll<<0.05\right)$ as shown in Fig 8 and correlated positively low $(\mathrm{r}=0.3715)$. The sound of 0 . tormota yielded a Protection index (PI) of $26.76 \%$ based on the number of mosquitoes that approached the blood meal, which was $7.36 \%$ below the startle response of 34.12 $\%$ for the sound of 0 . tormota in the same frequency range. The difference in protection index based on the number of mosquitoes that approached the blood meal determined through One-Sample T-test Statistics was significant $(\mathrm{p}=0.0247<0.05)$. 


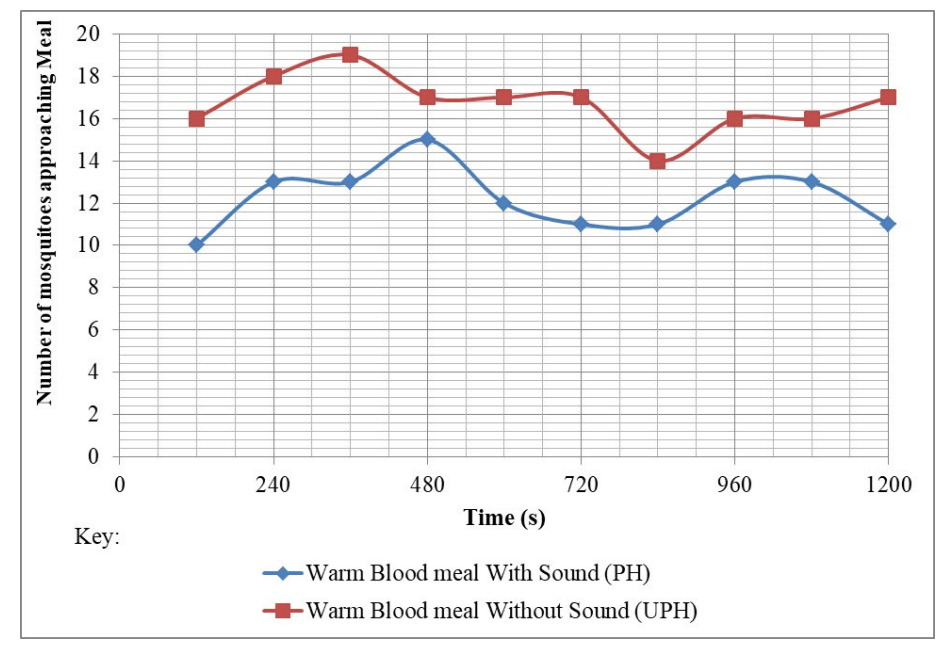

Fig 8: Number of mosquitoes approaching the control and treatment chamber for the sound of 0 . tormota

The number of mosquitoes that landed, probed and fed on the blood meal in the treatment chamber was lower compared to the number of mosquitoes that landed, probed and fed on the blood meal in the control chamber yielding protection index of $40.24 \%$ as given in Fig 9. The paired sample T-test comparison of the number of mosquitoes that landed, probed and fed on the blood meal in the treatment chamber to ones that landed, probed and fed on the blood meal in the control chamber showed a high significance difference with significance value $\mathrm{p}=1.9362 \times 10^{-5}$ with a strong positive correlation (Pearson's correlation value $\mathrm{r}=0.9599$ ). The protection index evoked by the sound of 0 . tormota was $6.12 \%$ higher than the reported startle response in female $A$. gambiae in the same frequency band by the sound of 0 . tormota based on behavioral responses, a difference which was not significant $(\mathrm{p}=0.2954)$ as determined through One-Sample T-test Statistics [55].

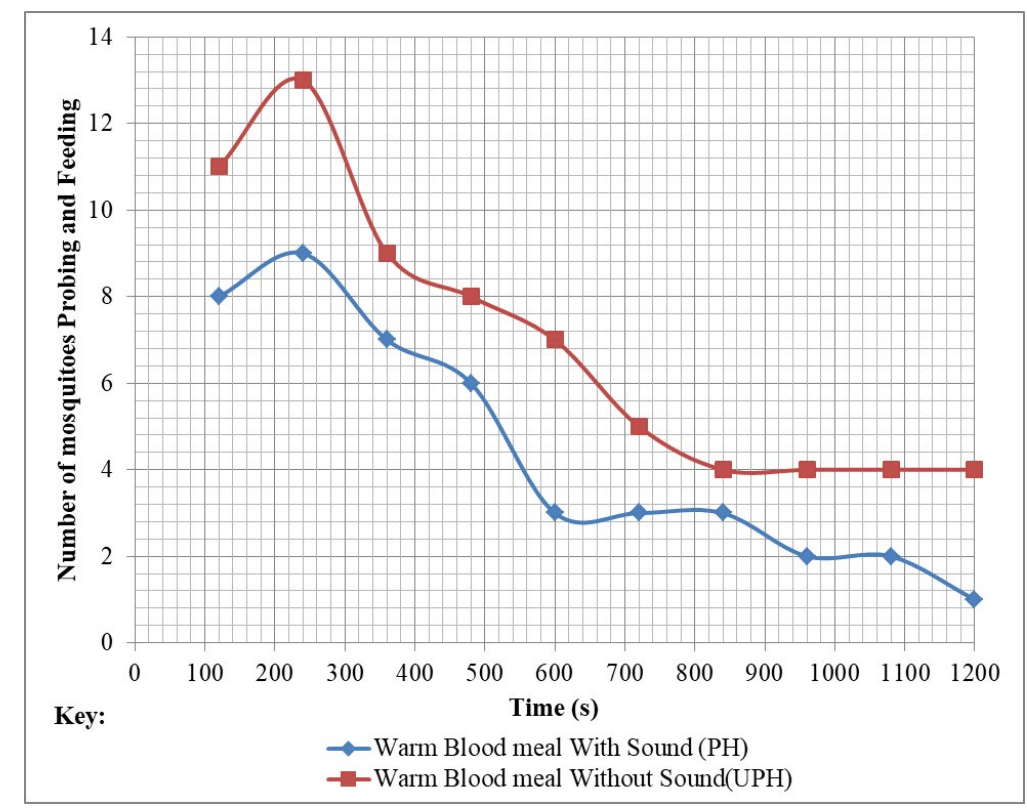

Fig 9: Number of mosquitoes feeding in the control and treatment chamber for the sound of 0 . tormota 
The protection index evoked by the sound of 0 . tormota exceeded the protection index due to the sound from the Anti-Pic $\AA$ (electronic mosquito repellency) by $9.94 \%$, a difference which was insignificant ( $p=0.1047$ $>0.05$ ) [4]. Also, the protection index due to the sound of 0 . tormota significantly exceeded the repellency due to the sound from an inbuilt ultrasonic device (AC-UD) in the knockdown tests with fan-off by $22.94 \%$ with $\mathrm{p}=0.0024<0.05$ [62]. Additionally, the protection index evoked by the sound of 0 . tormota was higher than the repellency determined from experiments with functioning electronic mosquito repellents (EMR) that mimicked calls from bats and male A. gambiae by $20.24 \%[4,15,27]$. The $10-34 \mathrm{kHz}$ sound of 0 . tormota was pulsated in nature with a minimum, maximum, and mean acoustic energy of $0.00025 \mathrm{~Pa}^{2} \mathrm{~s}, 6.74 \mathrm{~Pa}^{2} \mathrm{~s}$, and $0.44 \mathrm{~Pa}^{2} \mathrm{~s}$ respectively. The extent of disorderliness was 2.32 bits which were equal in aggregate entropy, average entropy, maximum entropy, and minimum entropy. Delta power $(\Delta \mathrm{P})$ of $22.40 \mathrm{~dB}$ was recorded in the $10-34 \mathrm{kHz}$ frequency range of the sound of 0 . tormota with a maximum and average power of $90.00 \mathrm{~dB}$ and $71.00 \mathrm{~dB}$ respectively as shown in Fig 10 and Table 1.

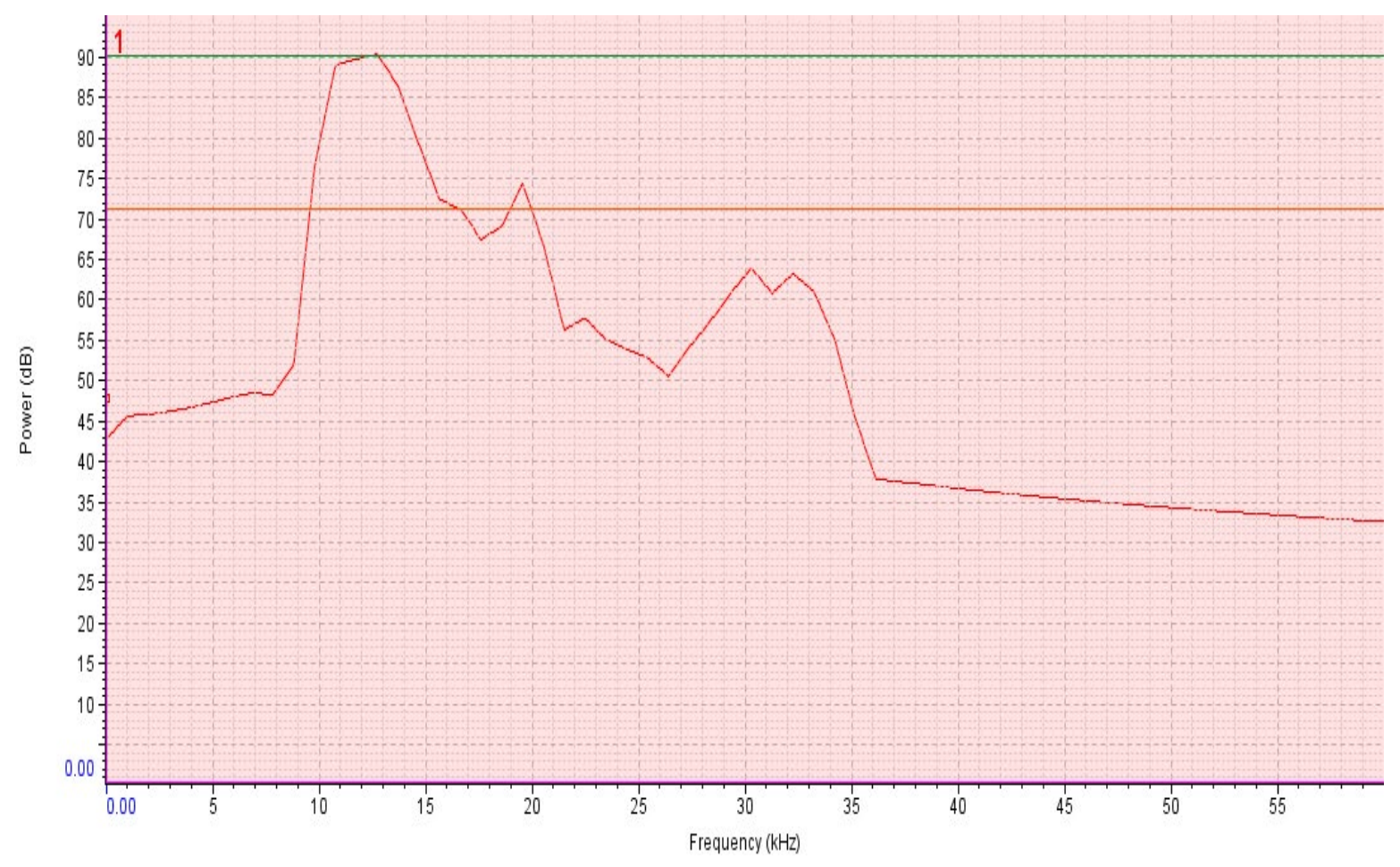

Fig 10: The power spectrum of the 10-34 kHz sound of 0 . tormota

The peak power was $90.00 \mathrm{~dB}$, equal to the maximum power recorded by the sound spectrum. There were 2,654 calls each lasting for a mean duration of $0.0439 \mathrm{~s}$ with mean measurements of $14.02 \mathrm{kHz}, 19.40$ $\mathrm{kHz}$, and 95.68 Pa for peak frequency (maximum entire), maximum frequency (maximum entire) and peak amplitude (mean entire) respectively for the sound of 0 . tormota. The horizontal green line and the Orange line showed the peak power and average power respectively in the $10-34 \mathrm{kHz}$ sound of 0 . tormota. The 2,654 calls recorded a maximum and mean call duration of $0.4198 \mathrm{~s}$ and $0.0439 \mathrm{~s}$ respectively. The mean and maximum measurements of the bandwidth (mean entire) were $5.29 \mathrm{kHz}$ and $16.90 \mathrm{kHz}$ respectively.

(ii). Determination and analysis of the landing rates and behavioral startle responses of the mated female $A$. gambiae on food attractant evoked by the 10-34 kHz sound of the male mosquito, $A$. gambiae

The control bioassay chamber and treatment bioassay chamber were connected to the feeding membrane 
through the netting. The food attractant on the treatment bioassay chamber was subjected to the $10-34 \mathrm{kHz}$ recorded sound of the male mosquito, A. gambiae. The female A. gambiae remained rested in the neutral chamber before deciding to move to the control or treatment bioassay chamber. The female A. gambiae were observed flying freely in and out of the control chamber. The minimum mosquito movement was observed in the control bioassay chamber. A total of six unfed female A. gambiae rested on the wall and the floor of the chamber. The female $A$. gambiae rested by projecting their abdomen in the air at about $45^{\circ}$. Fully fed mosquitoes rested on the wall and the floor of the control bioassay chamber. Mosquitoes in both the control bioassay chamber and treatment bioassay chamber rubbed their wings with their limbs to clean them. In the treatment bioassay chamber, the female A. gambiae were observed flying about, with one appearing disturbed. Two mosquitoes settled at the edge between the neutral chamber and the treated chamber after failing to land, probe and feed on the blood meal. One mosquito flew from the treatment bioassay chamber to the control chamber. Some mosquitoes were observed not to be fully fed. The number of mosquitoes inside the chamber, either the Control or treated chamber, were considered to have approached the blood meal. A comparison of the number of the female $A$. gambiae approaching meal in the control bioassay chamber exceeded the number of the female A. gambiae in the treatment bioassay chamber except at the $600^{\text {th }}$ second where the number of $A$. gambiae mosquitoes in both chambers were equal as shown in Fig 11. The equality in the number of the female A. gambiae approaching the blood meal in the control bioassay chamber and the treatment was attributed to the pulsate nature of the sound and weak acoustic energy and power during the $600^{\text {th }}$ second.

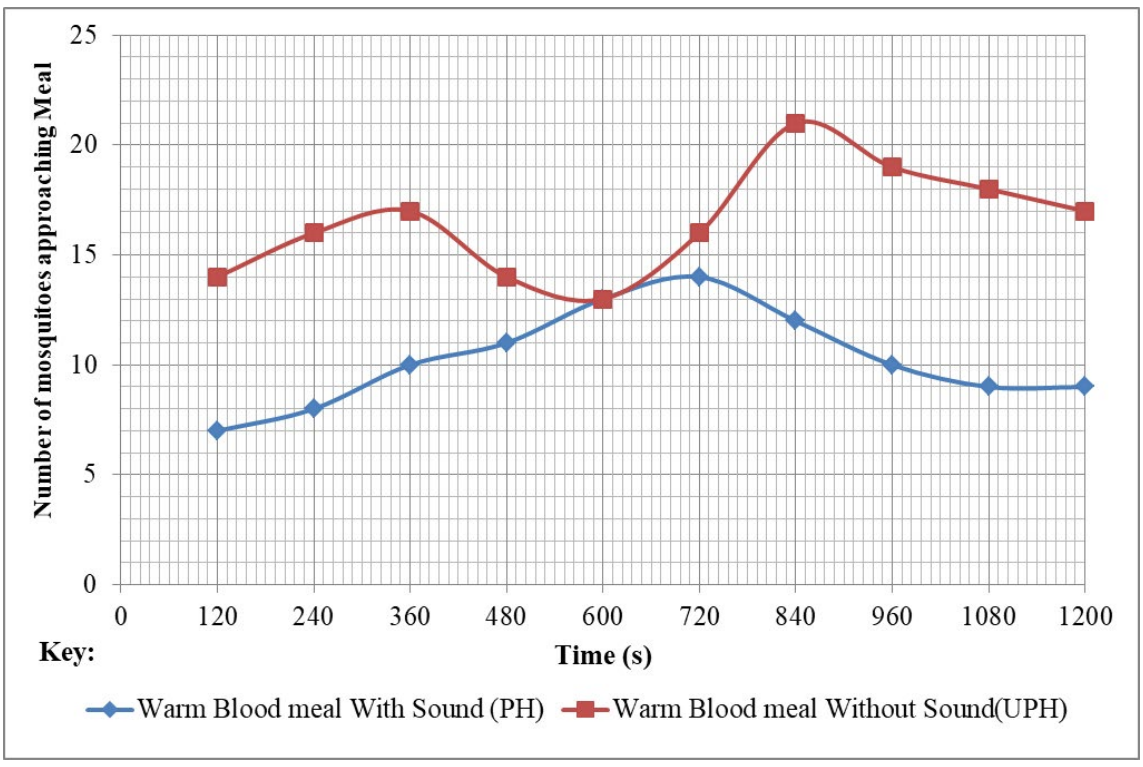

Fig 11: Comparison of the number of mosquitoes approaching the control and treatment chamber

The reduction in the number of female A. gambiae approaching the warm blood meal which was treated with the $10-34 \mathrm{kHz}$ sound of the male mosquito, A. gambiae was highly significant $\left(\mathrm{p}=2.1436 \times 10^{-4} \ll<0.05\right)$ and correlated positively low $(\mathrm{r}=0.0102)$. The protection index determined based on the number of mosquitoes that approached the respective blood meals evoked by the $10-34 \mathrm{kHz}$ sound of the male mosquito, A. gambiae was $36.24 \%$ which exceeded the repellency due to the $10-34 \mathrm{kHz}$ sound of 0 . tormota by $9.48 \%$. However, the difference in protection index due to the sound of the A. gambiae and protection index due to the sound of $O$. tormota determined in the study was not significant $(\mathrm{p}=0.1740>0.05)$ and correlated negatively low $(\mathrm{r}=-0.0235)$. The protection index due to the sound of the male A. gambiae was $2.12 \%$ above the reported startle response of $34.12 \%$ of to the sound of 0 . tormota in the same frequency band, a difference that was not significant $(\mathrm{p}=0.7209>0.05)$. Also, the protection index due to the sound of the male A. gambiae exceeded 
the repellency due to the sound from the Anti-Pic ${ }^{\circledR}$ (electronic mosquito repellency) by $5.94 \%$ though not significantly $(\mathrm{p}=0.3285>0.05)$ as determined through One-Sample T-test Statistics. The number of the mated female A. gambiae that landed, probed and fed on the blood meal in the treatment chamber was lower compared to the number of mosquitoes that landed, probed and fed on the blood meal in the control chamber yielding a protection index of $42.73 \%$ as given in Fig 12 .

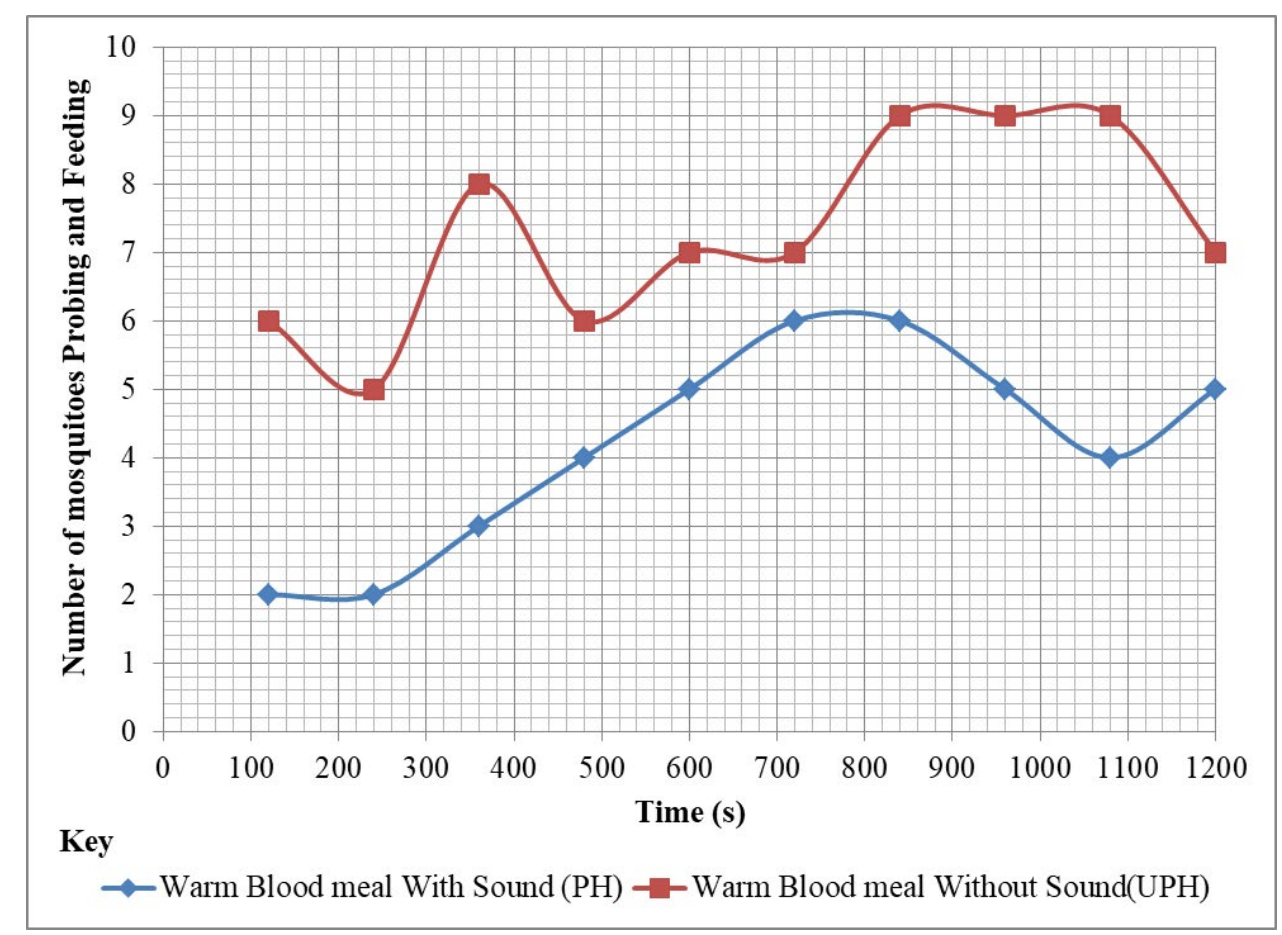

Fig 12: Number of mosquitoes feeding in the control and treatment chamber for the sound of the male $A$. gambiae

The paired sample T-test comparison of the number of mosquitoes that landed, probed and fed on the blood meal in the treatment chamber to the number of mosquitoes that landed, probed and fed on the blood meal in the control chamber indicated a high significance difference in repellency $\left(\mathrm{p}=5.3440 \times 10^{-5}<0.05\right)$ with a strong positive correlation (Pearson's correlation value $r=0.5522$ ). The insignificant difference between the protection index of the sound of $O$. tormota and the sound of the male A. gambiae ( $\mathrm{p}=0.1740>0.05)$ based on the number of mosquitoes that landed, probed and fed on the blood meal in both control and treatment bioassay chamber was $2.49 \%$ with the protection index of the sound of $O$. tormota being least. Also, the difference in repellency elicited by the sound of the male A. gambiae compared to the reported repellency of the sound of $O$. tormota which was based on behavioral responses only was not significant $(\mathrm{p}=0.1583>0.05)$ [55]. However, the difference in repellency due to the sound of the male A. gambiae was $12.43 \%$ higher than the claimed repellency due to the sound from the Anti-Pic ${ }^{\circledR}$ electronic mosquito repellent though the difference was not significant $(\mathrm{p}=0.0534>0.05)$ as determined through One-Sample T-test Statistics. Similarly, the protection index due to the sound of the male A. gambiae significantly $(\mathrm{p}=0.0014<0.05)$ exceeded the repellency due to the sound from an inbuilt ultrasonic device (AC-UD) in the knockdown tests with fan-off by $25.43 \%$ [62]. The minimally pulsate sound of the male A. gambiae in the $10-34 \mathrm{kHz}$ frequency range was $0.00014 \mathrm{~Pa}^{2} \mathrm{~s}, 0.92$ $\mathrm{Pa}^{2} \mathrm{~s}$, and $0.01 \mathrm{~Pa}^{2}$ s. Fig 13 represents the acoustic power spectra of the sound of male A. gambiae with the horizontal green line and the Orange line showing the peak power and average power respectively whose measurements are given in Table 1. The aggregate entropy, average entropy, average power, delta power $(\Delta \mathrm{P})$, maximum entropy, maximum power, minimum entropy and peak power in the slightly pulsate 10-34 kHz sound of male A. gambiae was 4.58 bits, 4.58 bits, $51.60 \mathrm{~dB}, 35.80 \mathrm{~dB}, 4.58$ bits, $64.50 \mathrm{~dB}, 4.58$ bits and 


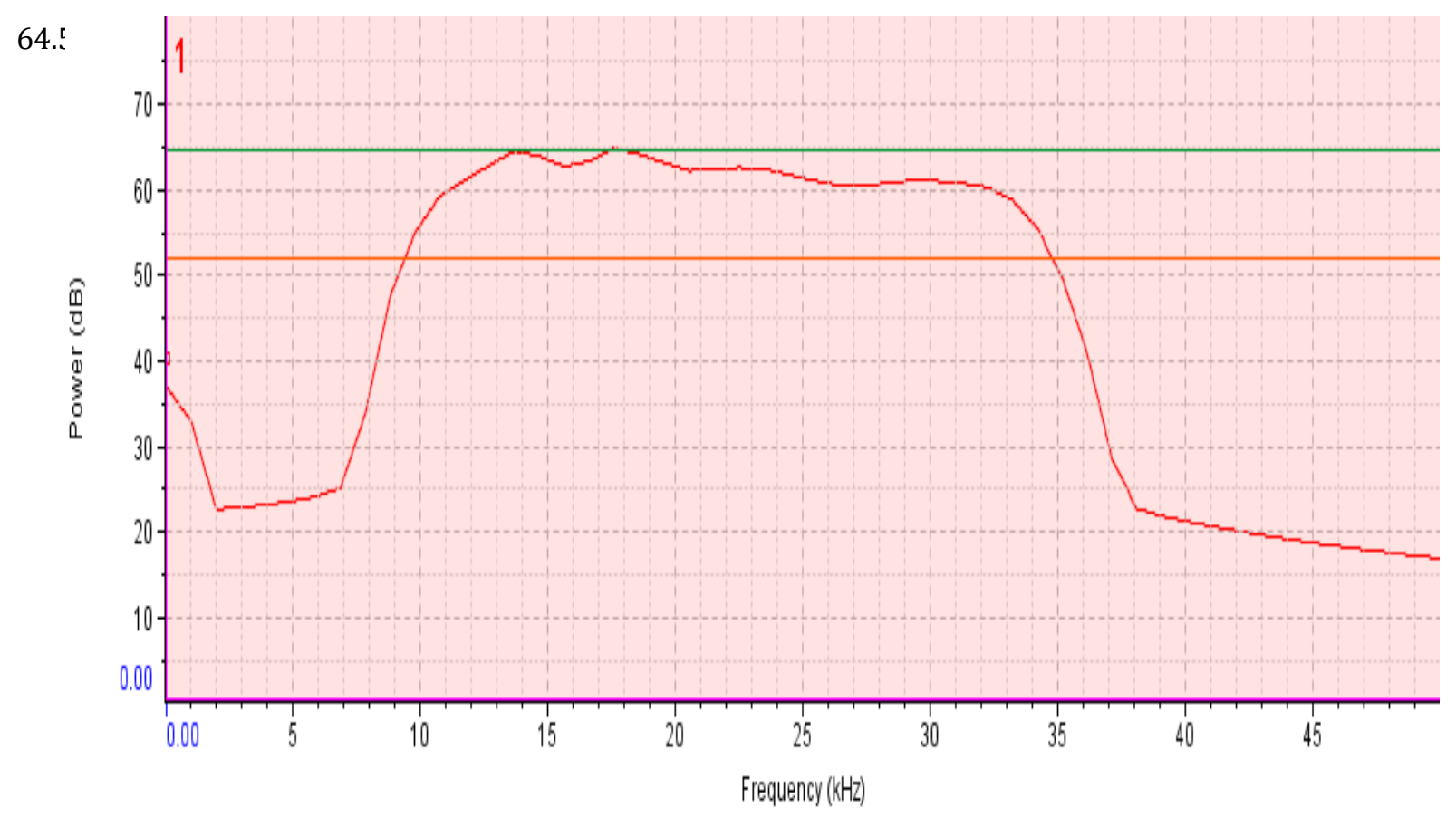

Fig 13: The power spectrum of the 10-34 kHz sound of the male $A$. gambiae

The maximum and mean of the bandwidth (mean entire) was $28.30 \mathrm{kHz}$ and $24.61 \mathrm{kHz}$. The difference in acoustic energy of the sound of the male mosquito, A. gambiae, and 0 . tormota was highly significant with significant values, $\mathrm{p}=4.4047 \times 10^{-50}<0.05$, and the parameters correlated positively low $(\mathrm{r}=0.0119)$. The sound of the male A. gambiae was composed of 41,135 calls whose maximum and mean duration was less than that of the sound of $O$. tormota by $0.1388 \mathrm{~s}$ and $0.0402 \mathrm{~s}$ respectively. The acoustic power of the sound of the $O$. tormota declined drastically compared to that of the sound of the male A. gambiae. However, the power deviation of the sound of the male A. gambiae exceeded the corresponding measurements for the sound of the $O$. tormota by $13.40 \mathrm{~dB}$. The wide power deviation, pulsate natured signal, enhanced entropy, many short duration calls, and wide bandwidth in the sound of the male A. gambiae evoked greater repellency compared to the sound of the 0 . tormota.

(iii). Determination and analysis of the landing rates, behavioral startle responses, and protection index in the mated female A. gambiae on food attractant evoked by the 10-34 $\mathrm{kHz}$ sound of D. leucas

The 10-34 kHz frequency band was filtered from the entire spectrum of the sound of D. leucas and used for the bioassay study. In the control bioassay chamber, the blood meal was not treated with sound. The mosquitoes flew about, to and from the neutral chamber with some unfed mosquitoes resting normally on the walls of the cage during the first $120 \mathrm{~s}$. Ten mosquitoes were observed feeding with no signs of disturbance. There was minimum movement by the mosquitoes in the neutral chamber after 240 s and two fully fed mosquitoes were observed resting on the chamber at the $480^{\text {th }}$ second. Limited movement, low activity was observed among the mosquitoes with one unfed mosquito rubbing its wings and extending its hind legs was observed in the chamber up to $1080^{\text {th }}$ second. During the last 120 s, two fully fed mosquitoes were seen resting in the control chamber.

In the treatment chamber, the mosquitoes were seen approaching the meal and landing and flying away along the wall, with others flying around the meal without successful landing during the first $120 \mathrm{~s}$. Between $120 \mathrm{~s}$ and 240 s, a limited movement among the mosquitoes was observed with on unfed mosquito flying into the 
chamber and resting on the wall of the chamber. During the 480-600s duration, two fully fed mosquitoes flew from the treatment bioassay chamber to the neutral chamber where it rested on the net. Also, one fully fed mosquito flew from the treated chamber to the control chamber then settled in the neutral chamber during the $600^{\text {th }}$ second. One unfed mosquito flew from the neutral chamber into the treated chamber but flew back to the neutral chamber immediately. Other unfed mosquitoes rested to the walls and floor with limited movement during the same duration. Bouncing along the net and on glass walls associated with knocks was observed during the 840-1200s duration.

The number of female A. gambiae approaching the blood meal in the control bioassay chamber was more compared to the number of the female A. gambiae in the treatment bioassay chamber except during the $240^{\text {th }}$ second where the number of mosquitoes in the treatment chamber exceeded the control as given in Fig 14 . Instances of attraction were confirmed during the $240^{\text {th }}$ second giving an instantaneous protection index of $-6.67 \%$ due to low acoustic energy and power and the least pulsate nature of the sound.

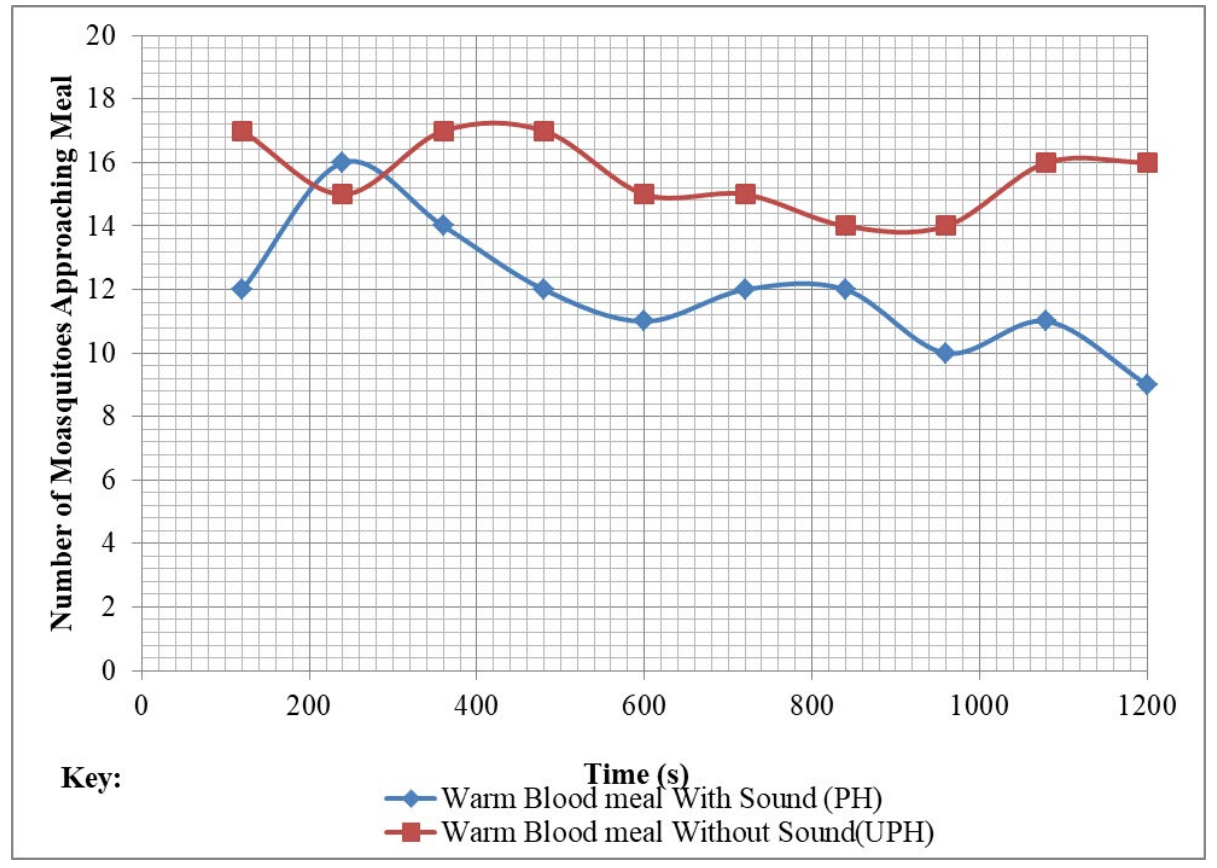

Fig 14: Number of mosquitoes approaching in the control and treatment chamber for the sound of $D$. leucas

The difference in the number of mosquitoes approaching the meal in the control bioassay chamber and the treatment bioassay chamber was highly significant $\left(\mathrm{p}=4.2749 \times 10^{-4}<0.05\right)$ and the parameters correlated positively low $(\mathrm{r}=0.1250)$. The overall protection index based on the number of mosquitoes approaching the blood meal in the control and treatment bioassay chambers was $23.43 \%$ which was less than the protection index for the sound of the male mosquito, A. gambiae and 0 . tormota by $12.81 \%$ and $3.33 \%$ respectively. Also, The protection index determined based on the number of mosquitoes that approached the respective blood meals evoked by the $10-34 \mathrm{kHz}$ sound of the male mosquito, A. gambiae was significantly ( $\mathrm{p}=0.0327<0.05$ ) lower than the protection index due to the $10-34 \mathrm{kHz}$ sound of $O$. tormota by $10.69 \%$.

The number of mosquitoes that landed, probed and fed on the blood meal in the treatment chamber was less than the number of mosquitoes that landed, probed and fed on the blood meal in the control chamber except during the $240^{\text {th }}, 840^{\text {th }}$ and $1080^{\text {th }}$ seconds where they were equal in number as given in Fig 15 . The number of 
mosquitoes that landed, probed and fed on the blood meal in the treatment chamber during the $960^{\text {th }}$ second exceeded the number of mosquitoes that landed, probed and fed on the blood meal in the control chamber.

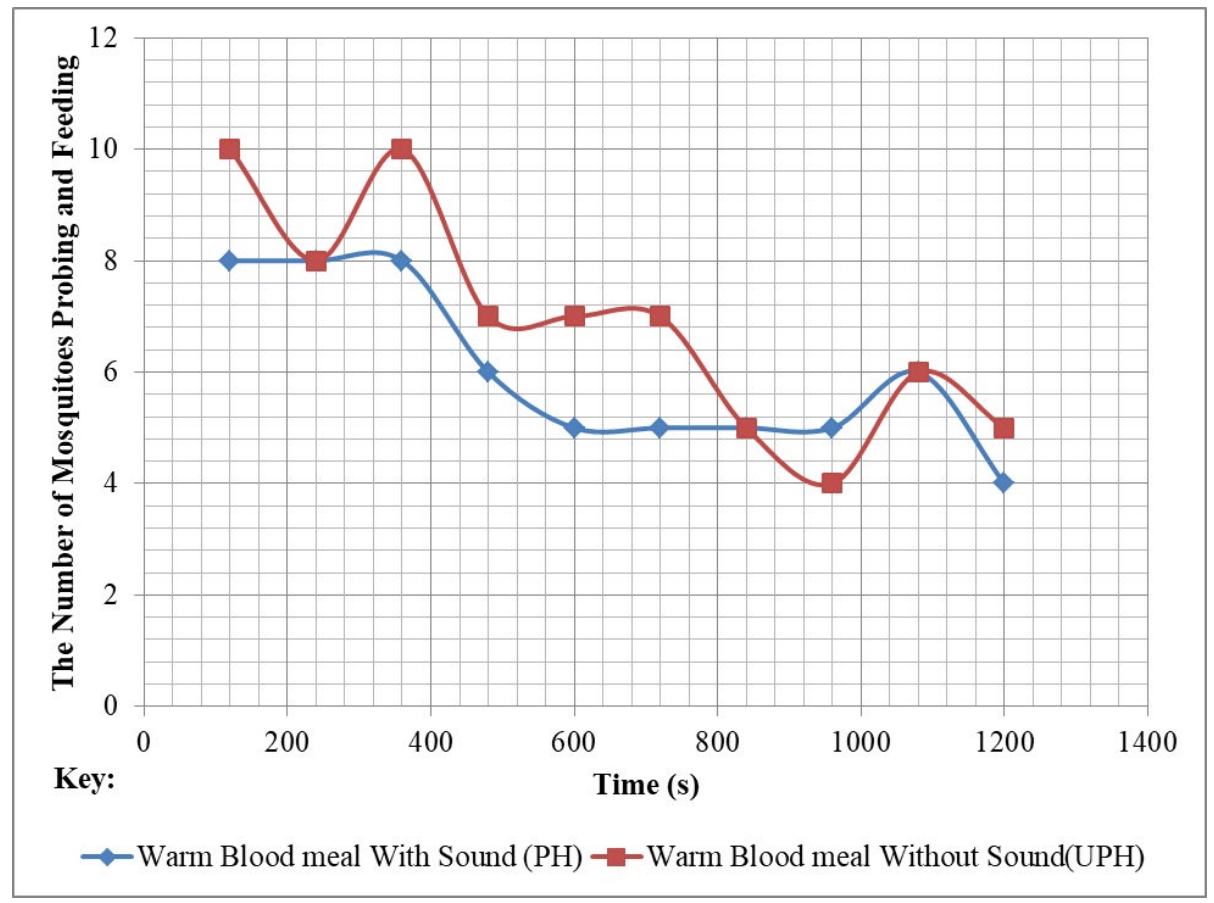

Fig 15: Number of mosquitoes feeding in the control and treatment chamber for the sound of $D$. leucas

The protection index based on the number of mosquitoes that landed, probed and fed on the blood meal in the treatment chamber and the number of mosquitoes that landed, probed and fed on the blood meal in the control chamber for the sound of D. leucas was $10.64 \%$, lowest by $32.09 \%$ and $29.60 \%$ from the protection index due to the sound of the male mosquito, A. gambiae, and 0 . tormota respectively. The paired sample T-test comparison of the number of mosquitoes that landed, probed and fed on the blood meal in the treatment chamber to the number of mosquitoes that landed, probed and fed on the blood meal in the control chamber for the 10-34 $\mathrm{kHz}$ sound of $D$. leucas showed a high significance difference in repellency ( $\mathrm{p}=0.02940<0.05$ ) with a strong positive correlation (Pearson's correlation value $r=0.8466$ ). The recorded sound of $D$. leucas yielded repellency which was $19.66 \%$ lower than the repellency due to Anti-Pic ${ }^{\circledR}$ electronic mosquito repellent [4]. A one-sample T-test comparison of the difference in the protection index due to D. leucas with the reported repellency due to Anti-Pic $\AA$ and the sound of $O$. tormota (based on behavioral responses) was highly significant with significance values, $\mathrm{p}=0.0049$ and 0.0016 respectively [4;55]. Also, the protection index due to the sound of $D$. leucas was insignificantly less than the repellency due to the sound from an inbuilt ultrasonic device (AC-UD) in the knockdown tests with fan-off by $6.66 \%$ with a significance value, $\mathrm{p}=0.2408>0.05$ [62]. The $10-34 \mathrm{kHz}$ sound of $D$. leucas recorded the least minimum acoustic energy of $0.0001 \mathrm{~Pa}^{2} \mathrm{~s}$ compared to the sound of the male $A$. gambiae and $O$. tormota. The maximum acoustic energy of the sound of $O$. tormota exceeded the acoustic energy of the sound of male A. gambiae and D. leucas by $5.82 \mathrm{~Pa}^{2} \mathrm{~s}$ and $3.53 \mathrm{~Pa}^{2} \mathrm{~s}$. The extent of disorderliness was 4.57 bits which were equal in aggregate entropy, average entropy, maximum entropy, and minimum entropy. Delta power $(\Delta \mathrm{P})$ of $28.50 \mathrm{~dB}$ was recorded in the $10-34 \mathrm{kHz}$ frequency range of the sound of 0 . tormota with a maximum and average power of $50.90 \mathrm{~dB}$ and $39.30 \mathrm{~dB}$ respectively as shown in Fig 16. 


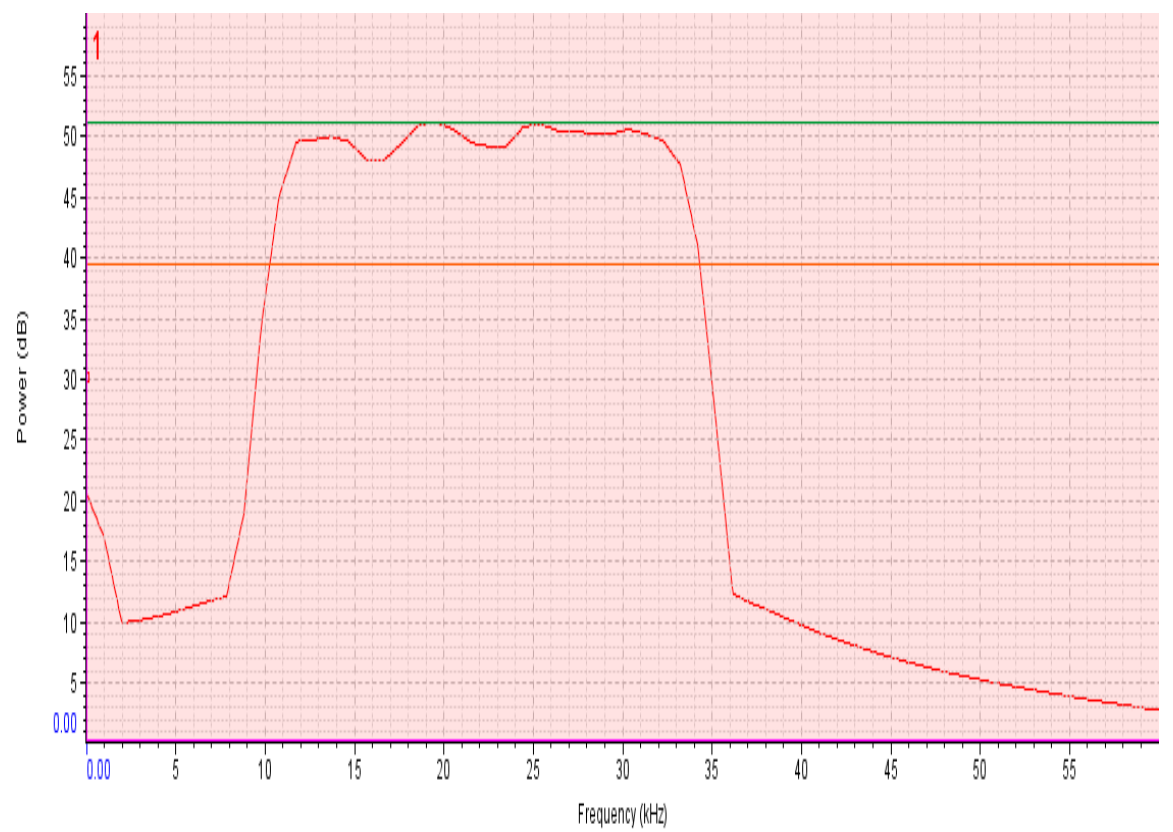

Fig 16:The power spectrum of the 10-34 kHz sound of $D$. leucas

The maximum and mean bandwidth (mean entire) in the $10-34 \mathrm{kHz}$ sound of $D$. leucas was $25.30 \mathrm{kHz}$ and $22.11 \mathrm{kHz}$ respectively which exceeded the corresponding parameters of the sound of $O$. tormota but less than the sound of the male A. gambiae. The call duration of the 15,299 calls lasted for a mean duration of $0.0013 \mathrm{~s}$. The 10-34 kHz sound of the male A. gambiae provided the highest protection index compared to other sounds studied as shown in Fig 17.

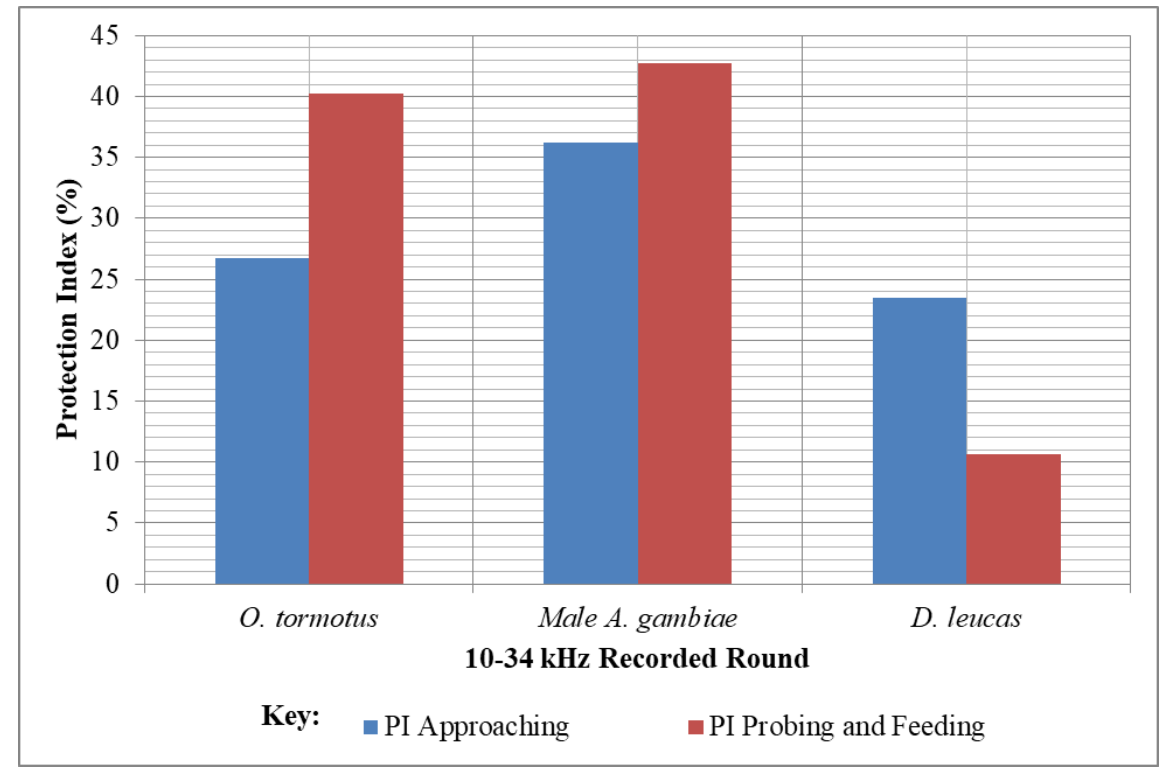

Fig 17: Comparison of the protection indices (PI) of animal sounds 
The pulsate and almost steady trend of the acoustic power in the sound of the male A. gambiae and the wide mean of the bandwidth (mean entire) provided superior parameters for the highest protection index and least landing rates as shown in Fig 17 and 18. Additionally, the entropy (disorderliness) of the sound of the male $A$. gambiae exceeded the entropy in the sound of the 0 . tormota and $D$. leucas by 0.01 bits and 2.26 bits respectively. The $10-34 \mathrm{kHz}$ frequency range for the sounds of the male A. gambiae, O. tormota, and D. leucas elicited 2.10 landings /minute, 2.20 landings/minute, and 3.00 landings/minute respectively. Also, the deviation in power was minimal within the same frequency range. Though the average power of the sound of 0 . tormota exceeded the corresponding average power of the sound of A. gambiae and D. leucas by $19.4 \quad 0 \mathrm{~dB}$ and $31.7 \mathrm{~dB}$ respectively. The sound of 0 . tormota was highly pulsated in nature with the greatest maximum acoustic energy and power. However, the steep slope in acoustic power, narrow bandwidth with the least entropy lowered the protection index of the sound of 0 . tormota in the 10-34 kHz frequency range. The ultrasonic components caused neural stress and refractory behavior to the male sound. However, the protection index due to the sound of the male A. gambiae was higher than the reported electronic mosquito repellent devices and that of $O$. tormota earlier investigated. The methodology employed in establishing repellency in female A. gambiae due to the sound of 0 . tormota in earlier studies differed with the current approach which is an improved methodology. The sound of $D$. leucas evoked the least repellency due to the minimal pulsate nature of the sound, low maximum and mean acoustic energy and low acoustic power.

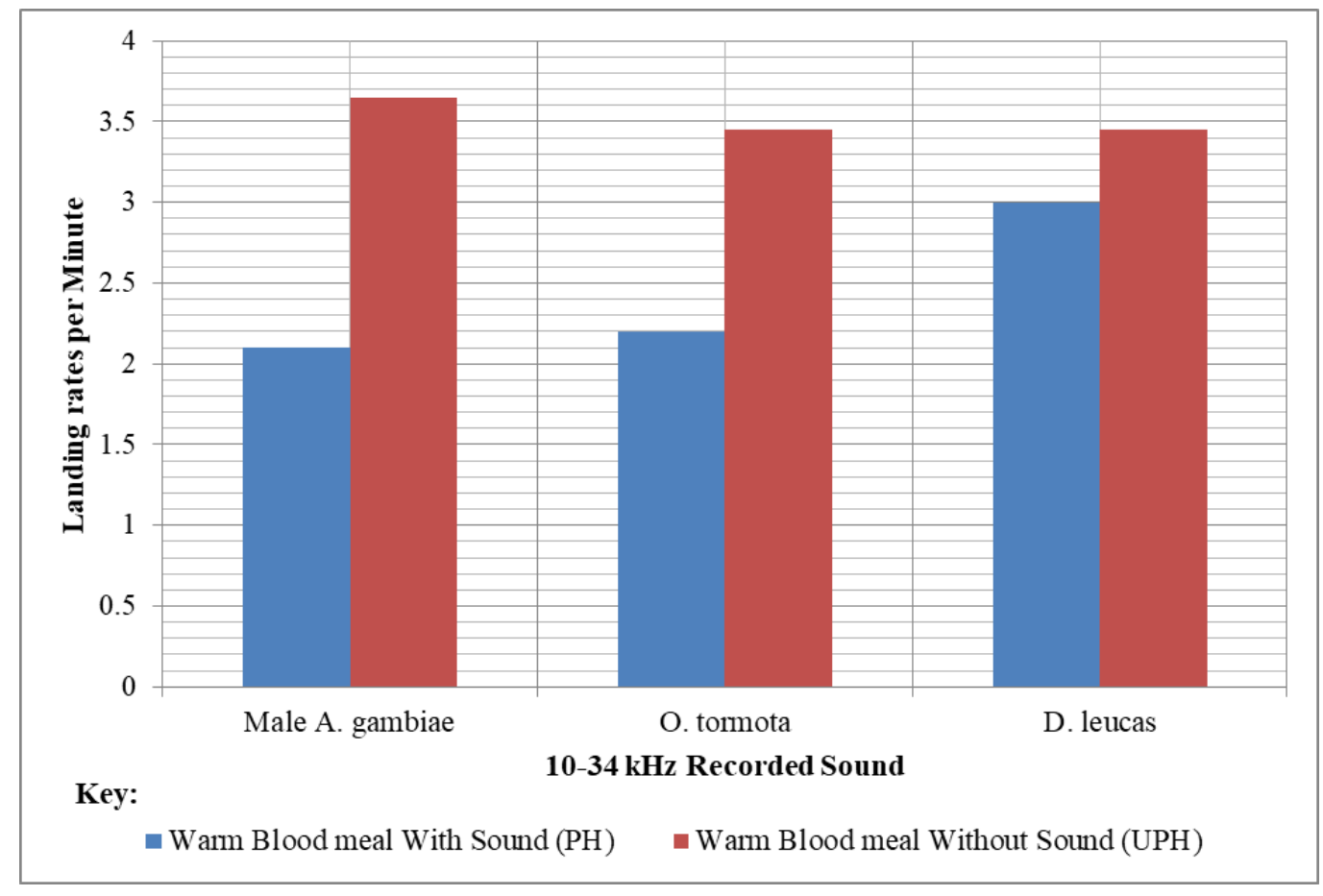

Fig 18: Comparison of the Landing rate evoked by Animal sounds

\section{CONCLUSION}

The sounds of the male A. gambiae, 0 . tormota, and D. leucas evoked different degrees of protection against the female A.gambiae with the sound of the male A. gambiae yielding the greatest protection index of 42.73 $\%$ with the least landing rate of 2.10 landings/minute. The high protection index was attributed to the pulsate and almost steady acoustic power, wide mean bandwidth (mean entire), and the minimal deviation between the maximum and minimum acoustic power within the $10-34 \mathrm{kHz}$ frequency range. 


\section{ACKNOWLEDGMENT}

We are greatly indebted to Egerton University (EU), Masinde Muliro University of Science and Technology (MMUST) and Kenya Medical Research Institute (KEMRI) for giving us the opportunity to conduct the research. Many thanks are extended to Prof. Feng, Raimund Specht of Avisoft Bioacoustics, Pettersson Elektronik AB, Cornell Lab of Ornithology, Bernard Agwanda of the National Museums (Kenya) and Prof. Herve Glotin of Institut Universitaire de France for their kind donations and technical support. This work was supported by the National Research Fund (NRF), Kenya through the award of the Doctorate Research Grant scholarship.

\section{REFERENCES}

1. Abdulrahman, H. M., Amoo, A. L., and Muhammad, B. U. (2019. Design and Construction of Electronic Pest Repellent for use in Homes and Farmland. Iconic Research and Engineering Journals. 3(1): 400-407. ISSN: 2456-8880

2. Aflitto, N. and DeGomez, T. Sonic Pest Repellents. The University of Arizona Cooperative, Extension. 2014. AZ1639: 1-4.

3. Antonelli, A., Murray, T and Danies, C. Pest Management for Prevention and Control of Mosquitoes with Special Attention to West Nile Virus. WSU-Puyallup. 2007: 1-7.

4. Andrade, C. F. S. and Bueno, V. S. Evaluation of Electronic Mosquito-Repelling Devices Using Aedes albopictus (Skuse) (Diptera: Culicidae). Neotropical Entomology. 2001; 30(3): 497-499

5. Arch, V. S., Grafe T. U. and Narins, P. M. (2008). Ultrasonic signaling by a Bornean frog. Biology Lett. 4: 19-22.

6. Arthur, B. J., Emr, K. S., Wyttenbach, R. A. and Hoy, R. R. (2014). Mosquito (Aedes aegypti) flight tones: frequency, harmonicity, spherical spreading, and phase relationships. The Journal of the Acoustical Society of America, 135(2), 933-941. https://doi.org/10.1121/1.4861233

7. Ashim, A. K., Tanveerul, H., Shaik, N., Farzand, A. and Parrmeshear, G. (2017). Design Considerations of Mosquito repellent unit. International Journal of Innovative Research in Technology. 3(9): 20-23

8. Barnard, R. D. Biological assay methods for mosquito repellents. Journal of the American Mosquito Control Association. 2005; 21 (4): 12-16.

9. Barnard, R. D., Bernier, R. U., Xue, R. and Debboun, M. (2006). Standard Methods for Testing Mosquito Repellents. Journal of the American Mosquito Control Association. 21(4):12-6. DOI: 10.2987/8756-971X(2005)21(12:BAMFMR)2.0.CO;2

10. Barredo, E. and DeGennaro, M. (2020). Not Just from Blood: Mosquito Nutrient Acquisition from Nectar Sources. Elsevier Ltd, Science Direct: Trends in Parasitology. 36( 5): 473-484. https://doi.org/10.1016/j. pt.2020.02.003 473

11. Baughman, T., Peterson, C., Ortega, C., Preston, S. R., Paton, C., Williams, J., Guy, A., Omodei, G., Johnson, B., Williams, H., O'Neill, L. S., Ritchie, A. S., Dobson, L. S. and Madan, D. (2017) A highly stable blood meal alternative for rearing Aedes and Anopheles mosquitoes. PLoS Negl Trop Dis 11(12): e0006142. https://doi. org/10.1371/journal.pntd.0006142

12. Bimbilé, S. N.S., Poda, B. S., Sawadogo, P. S., Gnankiné, O., Maiga, H., Fournet, F., Lees, R. S., Bouyer, J., Gilles, J., Sanon, A., Diabaté, A. and Dabiré, K. R.. (2018) Ecology of reproduction of Anopheles arabiensis in an urban area of Bobo-Dioulasso, Burkina Faso (West Africa): Monthly swarming and mating frequency and their relation to environmental factors. PLOS ONE. 13(11): e0205966. https://doi.org/10.1371/journal. pone.0205966 
13. Branstetter, B. K., DeLong, C. M., Dziedzic, B., Black. A. and Bakhtiari, K. (2016). Recognition of Frequency Modulated Whistle-Like Sounds by a Bottlenose Dolphin (Tursiops truncatus) and Humans with Transformations in Amplitude, Duration and Frequency. PLoS ONE. 11(2): e0147512. doi:10.1371/ journal. pone. 0147512.

14. Buescher, M. D., Rutledge, L. C., Wirtz, R. A. and Nelson, J. H. The dose-persistence relationship of DEET against Aedes aegypti. Mosquito News. 1983; 43: 364-366.

15. Center For the Advancement Of Health (CAH). "Electronic Mosquito Repellents Don't Work, Say Researchers." Science Daily. 2007. <www.sciencedaily.com/releases/2007/04/070417194230.htm>.

16. Centers for Disease Control and Prevention, CDC. (2010). Anopheles mosquitoes. Malaria. 1600 Clifton Rd. Atlanta, GA 30329-4027, USA 800-CDC-INFO (800-232-4636) TTY.

17.CDC. (2012). Anopheles mosquitoes. http://www.cdc.gov/ malaria/about/biology/mosquitoes/. Accessed 25 January, 2016.

18. Childs, L. M., Cai, F. Y., Kakan,i E. G., Mitchell, S. N., Paton, D., Gabrieli, P., Buckee, C. O. and Catteruccia, F. (2016). Disrupting Mosquito Reproduction and Parasite Development for Malaria Control. PLoS Pathogens. 12(12): e1006060. doi:10.1371/journal.ppat.1006060

19. Choumet V, Attout T, Chartier L, Khun H, Sautereau J, Robbe-Vincent A, et al. Visualizing Non Infectious and Infectious Anopheles gambiae Blood Feedings in Naive and Saliva-Immunized Mice. PLoS ONE. 2012; $7(12)$ : e50464. https://doi.org/10.1371/journal.pone.0050464

20. Combemale, P., Deruaz, D., Villanova, D. and Guilaumont, P. Les insectifuges ou les répellents. Ann. Dermatol. Venereol. 1992; 119: 411-434.

21. COSEWIC. (2014). Assessment and Status Report on the Beluga Whale Delphinapterus leucas St. Lawrence Estuary population in Canada. Catalogue No. CW69-14/703-2015E-PDF. ISBN 798-1-100-23284-3.

22. Dahalan, F. A., Churcher, T. S., Windbichler, N. and Lawniczak, M. K. N. (2019). The male mosquito contribution towards malaria transmission: Mating influences the Anopheles female midgut transcriptome and increases female susceptibility to human malaria parasites. PLoS Pathog. 15(11): e1008063. https:// doi.org/10.1371/journal.ppat.1008063

23. Day, J. F. (2016). Mosquito Oviposition Behavior and Vector Control. Insects. 7(4): 65. https://doi. org/10.3390/insects7040065

24. Diabaté, A., Yaro, A. S., Dao, A., Diallo, M., Huestisa, D. L. and Lehmann, T. Spatial distribution and male mating success of Anopheles gambiae swarms. BMC Evolutionary Biology. 2011; 11: 184.

25. EarthSky in Earth. How mosquitoes find you to bite you. Human World. Science Wire. 2015.

26. https://earthsky.org/earth/how-mosquitoes-find-you-to-bite-you. Accessed 28Th September, 2019.

27. Ellis, S., Franks, D.W., Nattrass, S., Currie, E. T., Cant, A. M., Giles, D., Balcomb, C. K. and Croft, P. D. (2018). Analyses of ovarian activity reveal repeated evolution of post-reproductive lifespans in toothed whales. Scientific Reports. 8, 12833 (2018). https://doi.org/10.1038/s41598-018-31047-8.

28. Enayati, A., Hemingway, J. and Garner, P. Electronic mosquito repellents for preventing mosquito bites and malaria infection (Review). The Cochrane Library Journal. 2010; 3: 1 - 16.

29. Enayati, A. and Hemingway, J. Malaria Management: Past, Present, and Future. Annual Reviews of Entomology. 2010; 55: 569-591.

30. Feng, S. A., Riede, T., Arch, V. S., Yu, Z., Xu, Z., Yu, X. and Shen, J. (2009). Diversity of the Vocal Signals of 
Concave-Eared Torrent Frogs (Odorrana tormota): Evidence for Individual Signatures. International Journal of Behavioural Biology. doi: 10.1111/j.1439-0310.2009.01692.x: 1-15

31. Frankel, A. S. (2009). Sound Production. Encyclopedia of Marine Mammals. Second edition. Academic press, London: 1056-1071.

32. Gething, P. W., Casey, D. C., Weiss, D. J., Bisanzio, D., Battle, K., Coates, M. M. and Hay, S. I. Mapping Plasmodium falciparum mortality in Africa between 1990 and 2015. The New England Journal of Medicine. Supplementary Information. 2016. 1:33.

33. Gibson, G., Warren, B., and Russell, I. J. (2010). Humming in tune: sex and species recognition by mosquitoes on the wing. Journal of the Association for Research in Otolaryngology : JARO. 11(4): 527-540. https://doi. org/10.1007/s10162-010-0243-2

34. Glotin, H. (2015). 'Beluga high velocity recordings'. SABIOD project.

35. Glotin, H. and Dolle, A. (2016). 'High Velocity bioacoustic an Anthropophony monitoring in Indian Ocean'. SABIOD - NortekMed project.

36. Godfray, H. C. J. Mosquito ecology and control of malaria. Journal of Animal Ecology. 2013; 82: 15-25.

37. Gonzales, K. K., \& Hansen, I. A. (2016). Artificial Diets for Mosquitoes. International journal of environmental research and public health. 13(12): 1267. https://doi.org/10.3390/ijerph13121267

38. Hadi, U. K., Koesharto, F. X. and Sigit, S. H. (2009). Study of the effect of ultrasonic device against the dengue mosquito , Aedes aegypti (Diptera: Culicidae). Prosiding Seminar Nasional Hari Nyamuk: 1 - 3.

39. Hanboonkunupakarn, B., \& White, N. J. (2016). The threat of antimalarial drug resistance. Tropical diseases, travel medicine and vaccines. 2(10): 2-5.

40.https://doi.org/10.1186/s40794-016-0027-8

41. Harwood, L. A., Norton, P., Day, B. and Hall, P. (2002). The harvest of beluga whales in Canada's western Arctic: Hunter-based monitoring of the size and composition of the catch. 55: 10-20.

42. Hoy, R. A boost for hearing in mosquitoes. Proceedings of the National Aacademy Sciences of the United States of America. 2006; 103 (45): 16619 -16620.

43. Ikeri, H. I., Onyia, A. I., Chima A.I. and Nwobodo A.N. (2017). Construction and Empirical Study of Electronic Piezzo Buzzer Mosquito Repellent. International Journal of Scientific \& Engineering Research. 8(11): 16051610. ISSN 2229-5518

44. Kaindoa, E. W., Ngowo, H. S., Limwagu, A., Mkandawile, G., Kihonda, J., Masalu, J. P., Bwanary, H., Diabate, A., \& Okumu, F. O. (2017). New evidence of mating swarms of the malaria vector, Anopheles arabiensis in Tanzania. Wellcome open research. 2: 88. https://doi.org/10.12688/wellcomeopenres.12458.1

45. Kent, R. J., and Norris, D. E. (2005). Identification of mammalian blood meals in mosquitoes by a multiplexed polymerase chain reaction targeting cytochrome B. The American journal of tropical medicine and hygiene. 73(2): 336-342.

46. Kou, H., Zhao. Y., Ren, K., Chen, X., Lu, Y. and Wang, D. Automated measurement of cattle surface temperature and its correlation with rectal temperature. PLoS ONE. 2017; 12(4): e0175377. https:// doi.org/10.1371/ journal.pone.0175377

47. Kremer, W. (2012). Ultrasound mosquito repellents: Zapping the myth. BBC World Service. https://www. bbc.com/news/magazine-20669080. Accessed 11th October 2019 at 10:15 am.

48. Kröber, T., Kessler, S., Frei, J., Bourquin, M. and Guerin, P. M. (2010). An in vitro assay for testing mosquito 
repellents employing a warm body and carbon dioxide as a behavioral activator. Journal of the American Mosquito Control Association. 26(4): 381-6. DOI:10.2987/10-6044.1

49. Kweka, E. J., Zhou, G., Munga, S., Lee, M. C., Atieli, H. E., Nyindo, M., Githeko, A. K. and Yan, G. Anopheline larval habitats seasonality and species distribution: a prerequisite for effective targeted larval habitats control programmes. Public Library of Science One. 2012; 7: 1-10.

50. Ladich, F. and Winkler, H. (2017). Acoustic communication in terrestrial and aquatic vertebrates. Journal of Experimental Biology. 220: 2306-2317 doi:10.1242/jeb.132944

51. Lapshin, D. N. and Vorontsov, D. D. (2018). Low-Frequency Sounds Repel Male Mosquitoes Aedes diantaeus N.D.K. (Diptera, Culicidae). ISSN 0013-8738, Entomological Review. 98(3): 266-271. DOI: 10.1134/ S0013873818030028

52. League, G. P., Onuh, O. C., and Hillyer, J. F. (2015). Comparative structural and functional analysis of the larval and adult dorsal vessel and its role in hemolymph circulation in the mosquito Anopheles gambiae. The Journal of experimental biology, 218(Pt 3), 370-380. https://doi.org/10.1242/jeb.114942

53. Lydersen, C., Martin, A. R., Kovacs, K. M. and Gjertz, I. (2001). Summer and autumn movements of white whales, Delphinapterus leucas, in Svalbard, Norway. Mar. Ecol. Prog. 219: 265-274.

54. Lyimo, I. N., Keegan , S. P., Ranford-Cartwright, L. C. And Ferguson, H. M. (2012). The impact of uniform and mixed species blood meals on the fitness of the mosquito vector Anopheles gambiae s.s: does a specialist pay for diversifying its host species diet? Journal of Evolutionary Biology. 25: 452-460. https://doi.org/10.1111/ j.1420-9101.2011.02442.x

55. McMeniman, C. J., Corfas, R. A., Matthews, B. J., Ritchie, S. A., \& Vosshall, L. B. Multimodal integration of carbon dioxide and other sensory cues drives mosquito attraction to humans. Cell. 2014; 156(5): 10601071. doi:10.1016/j.cell.2013.12.044

56. Mankin, R. W. Applications of acoustics in insect pest management. CAB Rev. 2012. 7(1):1-7 .

57. Mang'are, P. A., Maweu, O. M., Ndiritu, F. G. and Vulule, J. M. The Startling Effect of the Sound of C. afra and A. tormotus on the Female A. gambiae. International Journal of Biophysics. 2012; 2(3): 40-52

58. Marques, J., Cardoso, J.C.R., Félix, R.C., Power, D.M. and Silveira, H. A. (2020). Blood-Free Diet to Rear Anopheline Mosquitoes. Journal of Visualized Experiments. 155: 1-11. e60144, doi:10.3791/60144 (2020).

59. Maweu, O. M., Deng, A. L. and Muia, L. M. A Comparative study of A. gambiae male mosquito's response to frequency modulated [FM] and pulse modulated [PM] waves at different acoustic frequencies and distances. Indonesian Journal of Physics. 2009; 20: 81-84.

60. Mishima, Y., Morisaka, T., Itoh, M., Matsuo, I., Sakaguchi, A. and Miyamoto, Y. (2015). Individuality embedded in the isolation calls of captive beluga whales (Delphinapterus leucas). Zoological Letters . 1: 1-13.

61.https://doi.org/10.1186/s40851-015-0028-x

62. Mohankumar, D. Ultrasound and insects. Electronics and Animal Science. 2010. https://dmohankumar. wordpress.com/2010/04/08/ultrasound-and-insects/. Accessed on 1-November-17 10:00 PM.

63. Ogola, E., Villinger, J., Mabuka, D., Omondi, D., Orindi, B., Mutunga, J., Owino, V., \& Masiga, D. K. (2017). Composition of Anopheles mosquitoes, their blood-meal hosts, and Plasmodium falciparum infection rates in three islands with disparate bed net coverage in Lake Victoria, Kenya. Malaria journal. 16(1): 360. https:// doi.org/10.1186/s12936-017-2015-5

64. Okal, M. N., Lindh, J. M., Torr, S. J., Masinde, E., Orindi, B., Lindsay, S. W. and Fillinger, U. (2015). Analysing the oviposition behaviour of malaria mosquitoes: design considerations for improving two-choice egg count 
experiments. Malaria journal. 14(250): 1-17. https://doi.org/10.1186/s12936-015-0768-2

65. Okorie, P.N., Okareh, O.T., Adeleke, O., Falade, C.O. and Ademowo, O.G. Effects of an in-built ultrasonic device on Anopheles gambiae s.l mosquitoes in an indoor environment. International Research Journal of Engineering Science, Technology and Innovation (IRJESTI). (ISSN2315-5663) . 2015; 4(1): 5-11. DOI: http:/dx.doi.org/10.14303/irjesti.2015.074

66. Oliva, C.F., Benedict, M.Q., Lempérière, G. et al. (2011). Laboratory selection for an accelerated mosquito sexual development rate. Malar Journal. 10(135): 1-8. https://doi.org/10.1186/1475-2875-10-135

67. Parsons, C. and Dolman, S. (2004). The use of sound by cetaceans. Oceans of Noise. A WDCS Science report: 45-53.

68. Robert, D. and Jackson, J.C. (2006). Nonlinear auditory mechanism enhances female sounds for male mosquitoes. 103: 16619 - 16620.

69. Rodriguez, S. D., Drake, L. L., Price, D. P., Hammond, J. I., and Hansen, I. A. (2015). The Efficacy of Some Commercially Available Insect Repellents for Aedes aegypti (Diptera: Culicidae) and Aedes albopictus (Diptera: Culicidae). Journal of insect science (Online), 15(1), 140. https://doi.org/10.1093/jisesa/iev125

70. Rutledge, L. C., Wirtz, R.A., Buescher, M. D. and Mehr, Z. A. Mathematical models of the effectiveness and persistence of mosquito repellents. J. Amer. Mosq. Control Assoc.1985; 1: 56-62.

71. Saltin, B. D., Matsumura, Y., Reid, A., Windmill, J. .F, Gorb, S. N. and Jackson, J. C. (2019). Material stiffness variation in mosquito antennae. Journal of the Royal Society Interface. 16: 1-10. http://dx.doi.org/10.1098/ rsif.2019.0049

72. Simeon, M. I., Mohammed, A.S and Adebayo S. E. Development and preliminary testing of an electronic pest repeller with automatic frequency variation. International Journal of Engineering Science Invention. ISSN (Online): 2319 - 6734, ISSN (Print): 2319 - 6726. 2013; 2 (1): 14-20.

73. Simões, P. M. V., Gibson, G. and Russell, I. J. (2017). Pre-copula acoustic behaviour of males in the malarial mosquitoes Anopheles coluzzii and Anopheles gambiae s.s. does not contribute to reproductive isolation. Journal of Experimental Biology. 220: 379-385; doi: 10.1242/jeb.149757

74. Shen, J., Xu, Z., Yu, Z., Wang, S., Zheng, D and Fan, S. (2011). Ultrasonic frogs show extraordinary sex differences in auditory frequency sensitivity. Nature Communications. 2: 1-26. https://doi.org/10.1038/ncomms1339

75. Shen, J. X., and Xu, Z. M. (2016). The Lombard effect in male ultrasonic frogs: Regulating antiphonal signal frequency and amplitude in noise. Scientific reports. 6: 1-8. https://doi.org/10.1038/srep27103

76. Sumarnrote, A., Overgaard, H. J., Marasri, N., Fustec, B., Thanispong, K., Chareonviriyaphap, T., \& Corbel, V. (2017). Status of insecticide resistance in Anopheles mosquitoes in Ubon Ratchathani province, Northeastern Thailand. Malaria journal. 16(1): 299. https://doi.org/10.1186/s12936-017-1948-z

77. Thiévent, K., Hauser, G., Elaian, O. and Koella, J. C. (2019). The interaction between permethrin exposure and malaria infection affects the host-seeking behaviour of mosquitoes. Malar Journal. 18(79): 1-9. https://doi. org/10.1186/s12936-019-2718-x.

78. Tiwari, D. and Alam, M. (2016). Electronic Pest Repellent: A Review. 10.13140/RG.2.2.13557.78569.

79. Tizifa, T. A., Kabaghe, A. N., McCann, R. S., van den Berg, H., Van Vugt, M., \& Phiri, K. S. (2018). Prevention Efforts for Malaria. Current tropical medicine reports, 5(1): 41-50. https://doi.org/10.1007/s40475-0180133-y

80.Verma, S. S. (2017). Electronic Mosquito Repellent. Scientific India Magazine. https://scind.org/577/ Technology/electronic-mosquito-repellent.html. Accessed on 21st April, 2020 at $1452 \mathrm{hrs}$ 
81. World Health Organisation, WHO. World malaria report. 2011: 1-107.

82. World Health Organisation, WHO. World malaria report. 2013: 1-178.

83. World Health Organisation, WHO. World malaria report 2014: 1-157.

84. World Health Organisation, WHO. World malaria report. 2015: 1-148.

85. World Health Organisation, WHO. World malaria report: 2019: 4-11.

86. Yturralde, K. M. and Hofstetter, R. W. (2012). Efficacy of commercially available ultrasonic pest repellent devices to affect behavior of bed bugs (Hemiptera: Cimicidae). J Econ Entomol. 105(6): 2107-2114.

Citation: Mang'are P. A, et al. "Optimal Protection Index in Malaria Vector Host Elicited by the 10-34 kHz Animal Sounds". American Research Journal of Physics, vol 6, no. 1, 2020, pp. 1-29. DOI: 10.21694/ 2380-5714.20002

Copyright (c) 2020 Mang'are P. A, et al. This is an open access article distributed under the Creative Commons Attribution License, which permits unrestricted use, distribution, and reproduction in any medium, provided the original work is properly cited.

Received 02 July 2020 ; Accepted 21 July 2020; Published 2 July 2020

American Research Journal of Physics 